\title{
Hamstrings injury incidence, risk factors, and prevention in Rugby Union players: A systematic review
}

\section{C.D. Chavarro-Nieto, C.M Beaven, N.D Gill \& K. Hébert-Losier}

To cite this article: C.D. Chavarro-Nieto, C.M Beaven, N.D Gill \& K. Hébert-Losier (2021):

Hamstrings injury incidence, risk factors, and prevention in Rugby Union players: A systematic review, The Physician and Sportsmedicine, DOI: 10.1080/00913847.2021.1992601

To link to this article: https://doi.org/10.1080/00913847.2021.1992601 Accepted author version posted online: 12
Oct 2021.

Submit your article to this journal 준

LII Article views: 17

Q View related articles $\asymp$

View Crossmark data \lceil 
Publisher: Taylor \& Francis \& Informa UK Limited, trading as Taylor \& Francis Group

Journal: The Physician and Sportsmedicine

DOI: $10.1080 / 00913847.2021 .1992601$

Hamstrings injury incidence, risk factors, and prevention in Rugby Union players: A systematic review

${ }^{1}$ Chavarro-Nieto, C.D. ${ }^{1}$ Beaven, C.M; ${ }^{1,2}$ Gill, N.D; ${ }^{1}$ Hébert-Losier, K.

${ }^{I}$ Division of Health, Engineering, Computing and Science, Te Huataki Waiora School of Health, University of Waikato, New Zealand; ${ }^{2}$ New Zealand Rugby, Wellington, New Zealand.

${ }^{1}$ Division of Health, Engineering, Computing and Science

Te Huataki Waiora School of Health

University of Waikato

Adams Centre for High Performance

52 Miro Street, Mount Maunganui

3116 Tauranga, New Zealand

christian.chavarro@,gmail.com

kim.hebert-losier@waikato.ac.nz,

ORCID: 0000-0003-1087-4986

martyn.beaven@waikato.ac.nz

ORCID: 0000-0003-2900-7460

${ }^{2}$ New Zealand Rugby, Wellington

100 Molesworth Street, Thorndon, Wellington 6011New Zealand

nicholas.gill@,nzrugby.co.nz

*Corresponding author

E-mail: christian.chavarro@gmail.com

Telephone: +640225433011 
Hamstrings injury incidence, risk factors, and prevention in Rugby Union players: A systematic review

\section{ABSTRACT}

\section{Background}

Hamstring strain injuries are one of the most common injuries in Rugby Union, representing up to $15 \%$ of all injuries sustained. We aimed to systematically review and summarize the scientific literature that addressed hamstring strain injury incidence, risk factors, injury prevention or strengthening strategies, and strength or asymmetry measures in Rugby Union.

\section{Methods}

We conducted a systematic search to locate published peer-reviewed articles from PubMed, SPORTDiscus $^{\mathrm{TM}}$, Web of Science ${ }^{\circledR}$, and Scopus ${ }^{\circledR}$ e-databases. Studies included were original research conducted in Rugby Union that evaluated hamstring strength, hamstring strengthening interventions, and/or hamstring injury outcomes. Included studies were quality assessed using the Newcastle-Ottawa Scale.

\section{Results}

Twenty-four studies met inclusion and altogether involved 2866 participants. Isokinetic testing was the most common method used to quantify hamstring strength and imbalances in Rugby Union; with data indicating that professionals are stronger than amateurs, and forwards are stronger than backs. Regarding risk factors, we identified playing position, fatigue, previous injuries, between leg strength imbalances, lack of readiness to return to play post injury, and game actions (i.e., running). There is evidence to support the use of Nordic eccentric strength measures to inform practice, with strength and imbalances useful in 
predicting injuries. Strengthening programs with Nordic exercises significantly increased hamstring strength, increased musc 
le thickness, and decreased imbalance ratios in female and male players. A significant reduction in injury incidence and severity in professional players has been observed in players performing routines incorporating progressive Nordic exercises.

\section{Conclusion}

The aetiology of hamstring strain injuries is multifactorial, with playing position, fatigue, previous injuries, leg imbalances, lack of readiness to return to play, and running actions identified as contributing factors across levels. Combining strategies to prevent hamstring injuries and recurrences, and to inform return to play, is likely worthwhile and should include Nordic strength assessment and Nordic exercises.

\section{Keywords}

Hamstrings; strength; training; injuries; football.

\section{Introduction}

Hamstring strain injuries are one of the most common injuries in Rugby Union and represent 6 to $15 \%$ of all sustained injuries [1]. With respect to the epidemiology of hamstring injuries, acute hamstring strains have the highest recurrence rate of any muscle injury [2]. A previous hamstring injury is a paramount risk factor to sustaining another hamstring injury [3] and the elevated risk is proposed to be due to residual neuromuscular inhibition, strength deficits, altered muscle tendon morphology, and modified contractile mechanics [2].

The 2019 Rugby World Cup injury surveillance data revealed lower limb injuries accounted for almost $50 \%$ of all players absence days [4]. Hamstring strains were the second most common match injury after concussion, with hamstring injuries representing $9.8 \%$ of all 
match injuries and causing 467 missed days. The England Rugby Football Union reported that hamstring strains were their most common injury during training and the second most common injury during match play with 6.4 injuries per 1000 hours [5]. Moreover, the Welsh injury surveillance report from 2012 to 2016 recorded an increase in posterior thigh injury from 6.7 to 7.7 per 1000 hours, as well as 155.7 to 172.6 days lost during that time frame [6]. Hamstring strains are also of considerable concern at a younger school-age level, representing $21 \%$ of all injuries [7] and comprising $23 \%$ of all training time-loss in male amateur players.

Regarding prevention of sport-related injuries, a multifactorial approach is required and should involve the monitoring of intrinsic and extrinsic factors [8, 9]. For instance, asymmetries in strength between muscle groups and limbs are commonly assessed and used as screening methods in sports $[10,11]$. Strength imbalances between quadriceps and hamstrings have been linked to a four to five times greater risk of hamstring strain in football players, with an asymmetry in hamstring strengths between sides of $10-15 \%$ also considered to represent a risk for hamstring strains [12]. These imbalances appear more evident at slower angular velocities, and decrease at higher velocities [13]. Hamstring strength deficits and imbalances have been targeted with Nordic eccentric strengthening exercises, and a reduction of $51 \%$ in hamstring injury incidence across team sports was reported in a literature review and metanalysis of 8,459 athletes [14], including in Rugby Union [1].

Training and playing load variables, such as the number of high-speed running events, have been associated with hamstring injury occurrence in team sports and investigated mainly in football $[15,16]$. Although hamstring injuries often occur during the eccentric phase of running or kicking in rugby, similar to other team sports, severe hamstring strains can also 
occur because of specific-game related events in rugby, such as during tackles and competing for the ball on the ground [17]. In the tackling position, the hamstring is stretched fully, and the addition of a collision can further stretch the muscle, leading to a tendinous-junction tear. This mechanism of injury may be responsible for the increasing severity of hamstring injuries seen in Rugby Union in recent years [5], which is dissimilar to hamstring mechanisms in other sports. Thus, rugby demonstrates unique susceptibility and risk factors that require position-specific injury profiling to better target physical preparation and injury prevention strategies [18]. Given that player availability correlates with team success [19], research that targets injury prevention also has performance implications.

To prevent such injuries, it is important to identify how individual characteristics and game dynamics relate to contact and non-contact injury incidence. Therefore, we sought to critically examine and summarize the existing scientific literature on the topic of hamstring strain injuries in Rugby Union specifically. In particular, we aimed to systematically review and summarize the scientific literature that addressed hamstring strain injury incidence, risk factors, injury prevention and strengthening strategies, and strength or asymmetry measures in Rugby Union.

\section{Materials and methods}

\subsection{Information sources and search strategy}

We conducted a systematic review of the literature following the Preferred Reporting Items for Systematic Reviews and Metanalysis guidelines [20]. One author (CC) and one reviewer (CC) performed a systematic search on September $4^{\text {th }}, 2020$ to locate published peerreviewed articles from four electronic databases: PubMed, SciVerse Scopus, 
SPORTDiscus ${ }^{\mathrm{TM}}$, and Web of Science ${ }^{\circledR}$. The search strategy consisted of the following keywords and Boolean operators entered in the main search bar of each e-database: "hamstring AND rugby". The exact resulting search syntax in PubMed was: ("hamstring muscles"[MeSH Terms] OR ("hamstring"[All Fields] AND "muscles"[All Fields]) OR "hamstring muscles"[All Fields] OR "hamstring"[All Fields] OR "hamstrings"[All Fields]) AND ("football"[MeSH Terms] OR "football"[All Fields] OR "rugby"[All Fields]), SciVerse Scopus was: TITLE-ABS-KEY( hamstring AND rugby ), SPORTDiscus ${ }^{\mathrm{TM}}$ was: Boolean/Phrase: (hamstring AND rugby), and Web of Science ${ }^{\circledR}$ was: TOPIC: (hamstring AND rugby).

\subsection{Inclusion and exclusion criteria}

Articles were included when they were original, peer-reviewed research studies written in the English language, involved Rugby Union players, and included one or several of the following hamstring-related information: hamstring injury incidence, risk factors, strengthening or injury prevention programmes, and/or hamstring strength or asymmetry measures in Rugby Union players, regardless of sex, age and level of competition. Articles were excluded if these were not in the English language or did not involve Rugby Union players.

\subsection{Screening process}

Duplicates from the initial database search were removed first. Subsequently, two reviewers (CC, KHL) independently screened all remaining titles, abstracts, and full texts sequentially for inclusion. The study selection process was replicated for articles that were located through 
other sources (e.g. Google Scholar) and reference lists of included full-text articles. The two reviewers met to discuss any disagreements during the screening process and agreed on the articles to be included.

\subsection{Quality assessment}

Two reviewers $(\mathrm{CC}, \mathrm{IH})$ independently assessed the methodological quality of included studies $(\mathrm{n}=24)$ using the Newcastle - Ottawa Scale (NOS) [21]. The reliability of the NOS for case-control and cohort studies has fair to good inter-rater reliability and validity [22]. This tool was used as it is recommended by the Cochrane Collaboration [23], is a suitable alternative to other available tools to assess risk of bias [24], and could be used across the included studies. The NOS for case control and cohort studies was used across studies for ease of implementation and interpretation. Prior to assessment, the two reviewers met to discuss and familiarise themselves with the scales. All identifiable information (i.e., authors, affiliations, countries, and sources of publication) were removed from articles to reduce likelihood of assessment bias. Disagreements in the scores were resolved by discussion between the two reviewers, and consensus scores are presented in this article.

The tool uses a "star system", wherein more stars indicate a superior methodological quality and lower risk of bias. The NOS awards a maximum of 10 stars: five stars for selection (representativeness of the sample, sample size, non-respondents, and ascertainment of the exposure), two stars for comparability, and three stars for outcome (assessment of outcome and statistical test). Reviewers agreed that for the statistical test item, the highest star rating would be allocated for the reporting of confidence intervals, quartiles, or limits of agreement. The methodological quality of studies was divided into three groups based on the number of stars awarded: weak ( 0 to 3 stars), moderate (4 to 6 stars), and strong ( 7 to 10 stars) [25]. The 
design of each study was classified as cohort studies, case series, cases and controls, or randomized controlled trial.

\subsection{Data extraction and synthesis}

The first author (CC) extracted data from the selected full-text articles using a data extraction template customized to suit this review, and the last author (KHL) verified the data extracted. For each study, study design, participant information, level of competition, location of the study, study characteristics, assessment methods, and outcome data specific to hamstrings were extracted. Data were grouped and extracted under main themes of interest: (1) hamstring injury incidence and risk factors; (2) hamstring strengthening and injury prevention programmes; and (3) hamstring strength or asymmetry assessment methods and measures. Data were managed and analysed using Microsoft ${ }^{\circledR}$ Office Excel 2016 (Redmond, Washington). Conducting a meta-analyses on data was not considered given the aims of this review to summarize the existing literature, the high degree of heterogeneity of the data in the included studies, and the inappropriateness of pooling results from studies with different study designs, injury definitions, and outcome measures.

\section{Results}

\subsection{Included studies}

The flow diagram from the search strategy and screening process is shown Figure 1. Twentyfour studies met the eligibility criteria. 


\subsection{Study characteristics}

The main characteristics of the 24 studies that met inclusion are presented in Table 1. Based on Newcastle-Ottawa Scale NOS, most studies were of moderate quality ( $n=13,54 \%$ ) [2636]. Ten articles were of strong quality (40\%) [1,37-45], and one study was defined as weak quality $(6 \%)[46]$.

\subsection{Participants}

The 24 studies that met inclusion comprised of 2866 participants, male players were involved in 21 studies [1, 27-45, 47], one study involved female players [26], and two studies included both males and females [46, 48]. Eleven articles were conducted with professional players $[1,27,33,36-39,41,43-45]$, ten with amateur players $[26,28-32,34$, 42, 46-49], two with semi-professional players [40, 42], and not enough information about the level of competition was available in one study [35]. The articles were designed as cohort studies in 18 cases $(75 \%)[1,28,30,32,34,35,37-39,42-48]$, case series in three $(13 \%)$ [27, $31,33]$, case-controls in two (8\%) [26, 40], and a randomized control trial (4\%) [41]. Studies were conducted in the United Kingdom $(n=9)[1,26,29,36,38-40,42,43]$, Australia $(n=4)$ $[37,41,44,45]$, New Zealand $(n=3)[28,30,33]$, Ireland $(n=3)[27,32,48]$, USA $(n=2)[31$, 46], Morocco ( $\mathrm{n}=1)$ [47], Japan ( $\mathrm{n}=1)$ [34], and South Africa $(\mathrm{n}=1)$ [35] (Table 1).

\section{Figure 1.}

Table

1.

\subsection{Hamstring injuries: Incidence and risk factors}




\subsubsection{Professional level}

Hamstring strain injuries caused the greatest number of days of absence diagnosed in backs and was ranked third in forwards after shoulder and lumbar disc injuries [39]. In a two-part study involving professional players across two seasons, Part One monitored match injuries and Part Two monitored training injuries. In the first part, the incidence of hamstring strain injuries of backs were nearly three times greater than forwards [38]. Additionally, hamstring injuries were the most severe diagnosed injury in the backs and resulted in 17 days of absence. The lower limb was the most common anatomical site injured, with hamstring strains being the second most common injury in backs after thigh hematomas. Running was the most frequent mechanism of hamstring injury in game. In contrast to matches, forwards and backs had a similar incidence and mechanism of hamstring injuries during training [39], with more incidence of injuries during the preseason, with running again being the most common mechanism of injury, see Table 2.

Retrospective data analysis across five seasons in professional players indicated that $6 \%$ of all injuries were to the hamstring, with most hamstring injuries occurring during training and preseason [45]. Hamstring injuries occurred more whilst running, with similar incidence between forwards and backs. The median days lost for hamstring injury was 26 days, and most of the injuries were moderate $(60 \%)$, or severe $(37 \%)$. The running mechanics of 10 professional players were analysed. Injured players had greater ipsilateral thoracic lateral flexion, absorbed greater power at the knee, and had greater hip extension moment [44].

Brooks, Kemp [43] examined player injuries by playing position. Regarding severity of hamstring injuries, fly halves were the most affected players, followed by centres, wings, and 
the blind side flankers who presented with the most severe hamstring injuries whilst running. Data regarding hamstring injuries were also collected when implementing different hamstring training programs [1]. The incidence was similar between dominant and non-dominant legs. Overall, the most severe hamstring injuries happened during kicking. During matches, hamstring injuries were most frequent and severe in the last 20 minutes, and substitute players had twice as many injuries as starting players; however, both starters and substitutes had similar rates of recurrence.

Another study on hamstring strain injuries in professional players found that backs were the most affected players, with 21 days of absence overall [37]. The majority (45\%) of injuries were recurrences from the past season and $24 \%$ recurred in the same season. Most of the injuries involved the biceps femoris and occurred while running. Comparing the players with a recurrent injury, players with a previous hamstring injury in the last 12 months had 4.1 times higher risk of suffering a recurrent injury compared with players with no history of hamstring injury. Imbalances between limbs of more than $15 \%$ increased the risk of having a hamstring injury by 2.4 times; moreover, imbalances of more than $20 \%$ increased this figure to 3.4 times.

Regarding return to play after injuries, a five-week program to return to play with GPS technology was designed [27], and the recommendation to return to play after a hamstring injury was based on the ability to reach a running speed and intensity similar to pre-injury. The rehabilitation therapy recommended focusing on achieving maximum speed and long distances; involving cutting, passing, kicking, grappling, tackle drills, and wrestling; and achieving full training activities before returning to play. In one case study, a professional 
male Rugby Union player demonstrated an "abnormal" force-velocity profile during a 40-m sprint that resulted in an acute hamstring strain. The injurious sprint was characterised by an increase in horizontal force production compared to velocity when contrasted to his previous sprints and force-velocity profiles of his uninjured teammates [33].

\subsubsection{Semi-professional, amateur, and school level}

A community-level investigation across three seasons identified that hamstring strain injuries occupied the fifth place in terms of total injuries [42]. Running was the most frequent action of the game to produce hamstring injuries, and backs had a higher incidence than forwards. Hamstring injuries more frequently occurred in the first quarter of the game. At the amateur level, a prospective study with 65 players involving questionnaires found that $21 \%$ of all match and $30 \%$ of all training injuries were hamstring strain injuries [32].

With regards to the risk factors for hamstring injuries, three investigations have been conducted; the first followed semi-professional players with grade I hamstring strain injuries [40]. Their protocol comprised of two parts, one flexibility test and a slump test (validated for neural tension). In the group with a previous hamstring injury, $57 \%$ of the players had a positive slump test, whereas no positives were found in the control group. The results suggested that adverse neural tension should be assessed as a risk factor of hamstring injury and could be considered in return to play practices.

A prospective analysis of the relationship between motor imagery capacity of senior players (the ability to mentally perceive a rotated object) and hamstring injuries was undertaken [34]. Motor function was assessed with isokinetic hamstring quadriceps (H:Q) ratio, straight-leg 
raise angle, and a vertical jump test on one leg. Six players had symptoms of hamstring injuries (7 legs). The injured group (occurred in non-contact actions) had longer reaction times for the $0^{\circ}$ dorsal and $-90^{\circ}$ plantar views compared to the non-injured group (delay in 2.48 seconds) based motor imagery capacity test, with none of the motor function tests associated with injury. The third study in this area provided the option to players with previous hamstring injuries to wear or not wear thermal pants in training and matches during a season [35]. The incidence of injuries was significantly lower in players who chose to wear thermal pants.

Table 2.

\subsection{Hamstring strength and asymmetry assessment: methods and measures}

\subsubsection{Overview}

All methods and outcome measures used to assess hamstring strength are summarized in

Table 3. Isokinetic testing is considered the "gold standard" method to measure hamstring and quadriceps strength [50], with most of the studies assessing strength in rugby using isokinetic methods [26, 29-31, 41, 46, 47]. All of these isokinetic studies were conducted at $60 \%$, with a subset also using $180 \% \mathrm{~s}[29,31,47]$ and the one of poor methodological quality assessing at $35^{\circ} / \mathrm{s}$ [46]. Concentric hamstring strength was examined across all isokinetic studies, with five subjects considering eccentrics [29-31, 41, 46]. Nine studies measured the hamstring to quadriceps ratio (H:Q) [26, 28-31, 34, 36, 41, 46, 47] and five included dynamic control ratio (DCR) [29-31, 41, 46]. The H:Q is conventionally measured concentrically and used to quantify strength and imbalance between muscles and limbs. The dynamic control 
ratio is considered more functional, calculated as the eccentric hamstrings to concentric quadriceps strength ratio. There were no H:Q and DCR thresholds specifically established for Rugby Union players in the literature here reviewed. The Rugby Union literature cited thresholds from track and field that recommended H:Q values exceed 0.6 and DCR of 1.0 or above [51]. Other forms of hamstring strength testing methods used in rugby included eccentric [37], isometric [46], isotonic [31], and sphygmomanometer [36].

\subsubsection{Protocols}

\subsubsection{Isokinetic strength tests}

Concentric hamstring strength at $60 \%$ s ranged from 89 to $252 \mathrm{Nm}$ in amateur to professional players across positions and genders, and at $180^{\circ} / \mathrm{s}$ ranged from 71 to $121 \mathrm{Nm}$ in male amateurs (no data for professionals or females). Eccentric hamstring strength across at $60 \% \mathrm{~s}$ from $\sim 135$ to $220 \mathrm{Nm}$, and 209 to $220 \mathrm{Nm}$ at $180^{\circ} / \mathrm{s}$ in male amateurs (no data for professionals or females). Key findings were that professionals were stronger than academy [30], forwards were stronger than backs [28, 47]. In amateur players, experience had little effect on hamstring values [31] and effect of leg dominance was inconsistent across studies $[28,47][30]$.

In amateur players, $\mathrm{H}$ :Q values ranged from 0.45 to 0.56 , with no difference based on years of experience [31] or playing position [30]. Professional players displayed H:Q values of 0.52 to $0.68[28,41]$, with again no difference between playing positions [28], but the potential to increase with progressive Nordic eccentric training [41]. Concerning DCR values in amateur players, ratios ranged from 0.64 to 1.17 , and was significant greater in experienced players 
[31], but not affected by playing position or limb dominance [30]. DCR in professionals ranged from 0.74 to 0.81 [41], and was greater subsequent progressive Nordic eccentric hamstring training. Two studies undertook isokinetic knee strength assessment in amateur female athletes $[26,46]$, with one of these being of poor methodological quality. These studies identified that non-dominant limb H:Q (0.81) and DCR values were significantly greater than the dominant limb (0.74).

Deighan et al. [29] examined how conducting testing in seated vs supine influenced isokinetic knee extensor and flexor strength. The DCR at $180^{\circ} / \mathrm{s}$ and mean peak torque values seated were significantly greater than supine. Furthermore, knee extensors were stronger than flexors, and eccentric produced superior values than concentric. The study highlighted the significance of testing eccentric isokinetic strength with a hip flexion angle of approximately $10^{\circ}$ to determine imbalances and to screen for risk of injuries. Analysis in amateur players with both isometric and isokinetic strength tests in experienced and inexperienced player indicated experienced players had greater DCR at $60 \%$ values [31]. No significant differences were observed between players in DCR and H:Q at $180 \%$ s velocity, which the authors attributed to an adaptation to the high sprint demands of Rugby Union. At the same level of competition, Deighan et al. [29] found similar H:Q $60 \%$, but lower DCR values when compared to the results by Beyer et al. [31]

\subsubsection{Eccentric strength test with Nordic exercises}

Preseason Nordic hamstring eccentric testing performed with a load cell device [52], see Figure 2, indicated that weaker players (bilateral average $<267.9 \mathrm{~N}$ ) were at a similar risk of 
sustaining a hamstring injury than stronger players. Forwards were stronger than backs. Injured players demonstrated an imbalance between limbs of $17.4 \%$ that was significantly greater than injury-free players who displayed an imbalance of $\leq 10 \%$.

\section{Figure 2.}

Table 3.

\subsection{Hamstring strengthening programs}

During two seasons, 12 professional male teams followed a training program with stretching and strengthening exercises in one of three groups: 1) strengthening, 2) strengthening and stretching, and 3) strengthening, stretching, and Nordic exercises [1], see Table 4. The group performing Nordic exercises had a lower incidence of hamstring injuries compared to the group performing strengthening exercises alone. Although no significant differences were found in severity, there were less absence days in the Nordic exercise group. Second row players displayed the lowest incidence and severity of hamstring injuries.

In another study, two Nordic exercise 8-week training programs were examined: progressive and constant workload [41]. Along with the Nordic exercises, ultrasonography of the biceps femoris long head was performed. Both Nordic exercises strategies significantly increased the muscle thickness and length, without pennation angle changes. The progressive routine 
significantly increased the strength values by 7 to $8 \%, \mathrm{H}$ :Q from 0.53 to 0.57 , and DCR from 0.76 to 0.81 ; but not the constant workload group.

Intervention strategies with Nordic exercises have also been implemented in female rugby players [26]. Following a 10-week Nordic hamstring training intervention, isokinetic strength at $60 \%$ improved $11-13 \%$. Also of interest was that, following the training period, a significant reduction in bilateral strength imbalance from $10.3 \%$ to $4.6 \%$ was observed.

Table 4.

\section{Discussion}

\subsection{Hamstring injury incidence and risk factors}

We aimed to examine the scientific literature specific to Rugby Union and hamstring with focus on injury incidence, risk factors, injury prevention and strengthening strategies, and strength and asymmetry measures. The overall incidence of hamstring injuries during matches in professional players [38] was four times greater than community and amateur players [32, 42]. Across levels, backs suffered more hamstring injuries than forwards [1, $37,42]$ due to their greater running actions. The hamstring severity in community players was approximately 2 to 2.5 times higher than professionals [1, 37] suggestively due to lack of appropriate warm-up and hamstring training interventions in these cohorts [42]. 
In professional players, 23 to $45 \%$ of hamstring injuries were recurrences from either the current or previous season [37] [1], with a previous hamstring strain associated with a 4.1 times increased risk of recurrence [37]. The highest injury recurrence occurred in the first month of returning to play [1], suggesting that return to play was too quick and/or rehabilitation and reconditioning insufficient to meet the load demands. Similarly, $18 \%$ of the injuries have been found to recur in the first twelve days after returning to play and at the same site of the previous injury [35]. Of concern is that recurrences had more days lost (25 days) compared to new injuries (17 days) [1]. These studies altogether indicate that there is insufficient preparation before returning to training and playing after a hamstring injury, with a previous hamstring strain being a considerable risk factor. These findings align with a meta-analysis indicating that previous hamstring injury was a significant risk factor, in addition to other injuries (Anterior Cruciate Ligament, calf, and knee) for hamstring injuries [3]. Bourne et al. [37] highlighted the significant relationship between leg imbalances in eccentric strength and a previous hamstring strain injury in Rugby Union players, and concluded that players with a previous injury had an increased risk of sustaining another injury if they returned to play with pronounced strength imbalances between legs. In contrast, when isokinetic testing was included as a tool to return to play, van Dyk et al. [53] found no value in this strength measure as a criterion for return to play after a hamstring injury in football players. Whatever the tool used, rugby players should be monitored in their return to play progression and assessed periodically for imbalances, especially if they have sustained a previous hamstring injury.

Running was the most common action of the game to produce a hamstring strain injury across levels, accounting for 68 to $85 \%$ of all injuries in professionals $[1,37,45]$ and $54 \%$ in amateur [42]. There was somewhat conflicting evidence in terms of when in the game 
injuries were more likely to occur. In professionals, the majority of the injuries in backs occurred in the last part of each half, but incidences were greater later in the game in forwards [1]. In contrast, hamstring injuries occurred more frequently in the first quarter in community players[42]. These contrasting results suggest insufficient warm-up or game preparation at lower levels of competition as a potential contributor to injury rates, whereas fatigue may be a greater contributor at higher levels. This proposition relating to warm-up strategies is indirectly supported by findings of a significant decrease in hamstring injuries when previously injured players wore warm pants during training and matches [35].

Running training and high speed exposures have been postulated as an effective tool to reduce hamstring injuries [54]. One of the recommendations by Buckthorpe et al. [15] for preventing hamstring injuries involved incorporating high speed-running routines at least twice a week at $95 \%$ of maximum speed. Analysis of a player with a hamstring injury identified impaired sprint accelerations with a decrease in horizontal force production, potentially due to weak hamstring or gluteal muscles, before the injury and after return to play [33]. The authors highlighted the importance of running activities for preventing hamstring injuries, and suggested sprint time measurement to detect deficits during the initial acceleration phase. Magnetic resonant imaging of hamstring injuries in professional players showed the biceps femoris long head fascicle was the most injured muscle $(73 \%)$, and that this injury occurred most frequently in running actions $(77 \%)$ [45]. In contrast to football players who sustained more Proximal Myofascial junction intramuscular injuries [55], the Distal Myofascial junction site was more common in Rugby Union players, re-emphasising the importance of Nordic exercises to target this portion of the muscle [56]. 


\subsection{Hamstring strength}

Playing level and position in rugby have previously been associated with different attributes and demands, including body composition, speed, strength, power, and repeated sprint performance [57]. The isokinetic measures here reviewed support these findings overall, with professionals being stronger than amateurs and forwards being stronger than backs $[30,28]$. The differences between playing positions and levels likely relates to the match demands of forwards requiring greater leg strength (i.e., tackling, scrummaging, rucking, mauling, and pick-and-goes) than backs, and strength increasing with years of experience and the greater demands at higher levels.

From the literature reviewed, no H:Q and DCR thresholds from isokinetic testing have been established as optimal or protective of injury for Rugby Union. In track and field, $\mathrm{H}: \mathrm{Q}$ values exceeding 0.6 [51] and DCR values of 1.0 or above are considered normal [59] and recommended for reducing the risk of hamstring injuries [51]. H:Q values below 0.6 have been shown to increase hamstring injuries 17-fold in sprinters [10]. Across the Rugby Union literature reviewed, H:Q values were typically lower than $0.6[30,31][42]$, although reported to surpass 0.6 in professional players [28]. Similarly, DCR were often below $1.0[28,31,41]$, even in professionals. Addressing these imbalances could potentially reduce the relatively high incidence of hamstring strains in Rugby Union. That said, van Dyk et al. [60] found no relationship between H:Q isokinetic strength ratios in injured and uninjured football players. Furthermore, the systematic review and metaanalysis for hamstring strain injury risk factors by Freckleton, Pizzari [3] did not find evidence that any isokinetic ratio, at any speed or type of contraction, was associated with 
the risk of hamstring injuries. Therefore, it remains uncertain whether improving these ratios would actually reduce hamstring incidence in rugby.

In amateur female players, an increase in strength was found, as well as a reduction in imbalance between limbs, after a Nordic exercise intervention [26]. A systematic review by Hewett et al. [61] found no significant difference between females and males in isokinetic H:Q values at slow velocities; although males had significantly greater H:Q values at higher angular velocities (from 30 to $360^{\circ} / \mathrm{s}$ ). The reduced capacity of females to control the knee joint at high velocities might increase their risk to sustain a hamstring injury during high speed running [62]. These results suggest that assessing H:Q values at higher angular velocities might be more clinically-relevant in the context of Rugby Union and hamstring injuries, and should be used preferentially by clinicians in the context of assessing injury risk.

Nordic eccentric strength assessments are proposed as a more feasible, physiological, and functional test than isokinetic. Forces generated during Nordic exercises are similar to those observed during sprinting [63]. However, only one study measured Nordic eccentric values in Rugby Union. Bourne et al. [37] detected significantly weaker limbs in injured than uninjured limb, as well as greater imbalances between legs in injured than uninjured players. Imbalances of more than $15 \%$ and $20 \%$ between legs increased the risk of hamstring injuries by 2.4 and 3.4 times, respectively. A review by Kalkhoven et al. [64] found that during all the phases of sprinting, the hamstring muscles are active (from the stance phase to the swing phase). The "hamstring muscle slack", a term which suggests the hamstring act isometrically in the late swing of sprinting, does not occur. These authors concluded that the hamstring contracts in an eccentric fashion in the late swing 
phase, such that an eccentric test may be a more appropriate way to measure hamstring strength and capability. This interpretation of the kinematics of sprinting may also suggest that eccentric exercises are a better way to train hamstring function. That said, authors of a systematic review and meta-analysis of different devices measuring hamstring eccentric strength in different sports with the Nordbord device cautioned when assessing hamstring peak strength and imbalances to estimate hamstring injury risks [65]. This caution was due to the Nordic hamstring test not eliciting the same demands as running, and therefore, it should be used as the sole tool to assess injury risk in-season neuromuscular status [65].

\subsection{Hamstring strengthening programs}

Rugby Union players that followed a Nordic eccentric exercise program exhibited significantly improved hamstring strength [26, 41], decreased bilateral strength imbalances [26], and reduced hamstring injury incidence and severity (i.e., less days absent from training and matches) [1]. Interventions also improved H:Q and DCR values, as well as an increased the biceps femoris long head fascicle length and thickness. As a short fascicle length has been shown to increase the risk of hamstring injuries, and longer fascicles identified as a protective factor of hamstring strain injuries in older football players with previous hamstring injuries [66], the progressive Nordic program in Rugby Union can promote beneficial morphological adaptations. Giakoumis [67] suggested a number of possible mechanisms underpinning the beneficial effects of Nordic exercises in athletes other than semitendinosus and biceps femoris fascicle lengthening, including hypertrophy of these muscles and synergistic role of the semitendinosus in sprinting activities. Compared to other exercises, eccentrics elicit greater electromyographic 
activity, increases in strength, and muscle adaptations [67]. Therefore, although cautioned against being used as a sole screening measure [65], the integration of Nordic exercises appear of benefit to Rugby Union and are recommended. The intervention programs in Rugby Union with beneficial effects followed similar training strategies over the course of 8 to 10 weeks, and can be recommended to practitioners. These programs involved eccentric hamstring training 2 to 3 times a week, completing 3 to 4 sets of 6 to 10 repetitions.

\section{Conclusion}

Current literature is lacking to support the evidence-based use of isokinetic strength, H:Q, and DCR measures to inform injury prevention and return to play strategies for hamstring injuries in Rugby Union. Nordic eccentric strength assessment has been shown to be a better physiological and functional test, with differences in strength and imbalances predicting new and recurrent injuries. Strengthening programs with Nordic exercises significantly increased hamstring strength measures, decreased imbalance ratios, and reduced injury incidence and severity. The aetiology of hamstring injuries is multifactorial, with playing position, fatigue, previous injuries, leg imbalances, lack of readiness to return to play, and running actions identified as contributing factors across levels. Combining strategies to prevent hamstring injuries and recurrences, and to inform return to play, is likely worthwhile and should include Nordic strength assessment and Nordic exercises. It has been proposed that high-speed running and warm-up routines may be important in the prevention of hamstring injuries, although strong evidence for these suggestions is lacking. 


\section{References}

1. Brooks JH, Fuller CW, Kemp SP, Reddin DB. Incidence, risk, and prevention of hamstring muscle injuries in professional rugby union. American Journal Sports Medicine. 2006;34(8):1297-306. doi: 10.1177/0363546505286022.

2. Brukner P, Khan K. Brukner \& Khan's Clinical Sports Medicine: Injuries. McGraw-Hill Education Australia; 2016.

3. Freckleton G, Pizzari T. Risk factors for hamstring muscle strain injury in sport: a systematic review and meta-analysis. British Journal of Sports Medicine 2013;47(6):351-8. doi: 10.1136/bjsports-2011-090664.

4. Fuller, Taylor A, Douglas M, Raftery M. Rugby World Cup 2019 Injury Surveillance Study. South African Journal of Sports Medicine. 2020;32(1). doi: 10.17159/2078$516 \mathrm{X} / 2020 / \mathrm{v} 32 \mathrm{i} 1 \mathrm{a} 8062$.

5. RugbyFootballUnion, Surveillance. Data from the 2017/18 Professional Rugby Injury Surveillance Project (PRISP). In: Group AbtEPRISPS, editor. 2017-2018 Season Report. https://www.englandrugby.com/mm/Document/General/General/01/33/22/57/InjurySurveilla nceReport2017-18_English.pdf: England Rugby; 2019. p. 53.

6. Bitchell CL, Mathema P, Moore IS. Four-year match injury surveillance in male Welsh professional Rugby Union teams. Physical Therapy in Sport. 2020;42:26-32. doi: https://doi.org/10.1016/j.ptsp.2019.12.001.

7. Group IRISIPS. School Senior \& Junior Cup Rugby 2019 - 2020 Season Report. The Irish Rugby Injury Surveillance Project: University of Limerick; 2020. 
8. Opar DA, Williams MD, Shield AJ. Hamstring strain injuries: factors that lead to injury and re-injury. Sports Medicine. 2012;42(3):209-26. doi: 10.2165/11594800-00000000000000.

9. Orchard J, Marsden J, Lord S, Garlick D. Preseason hamstring muscle weakness associated with hamstring muscle injury in Australian footballers. The American Journal of Sports Medicine. 1997;25(1):81-5. doi: 10.1177/036354659702500116.

10. Yeung SS, Suen AM, Yeung EW. A prospective cohort study of hamstring injuries in competitive sprinters: preseason muscle imbalance as a possible risk factor. British Journal of Sports Medicine. 2009;43(8):589-94. doi: 10.1136/bjsm.2008.056283.

11. Knapik JJ, Bauman CL, Jones BH, Harris JM, Vaughan L. Preseason strength and flexibility imbalances associated with athletic injuries in female collegiate athletes. The American Journal of Sports Medicine. 1991;19(1):76-81. doi: $10.1177 / 036354659101900113$.

12. Croisier JL, Ganteaume S, Binet J, Genty M, Ferret JM. Strength imbalances and prevention of hamstring injury in professional soccer players: a prospective study. The American Journal of Sports Medicine. 2008;36(8):1469-75. doi: $10.1177 / 0363546508316764$

13. Wrigley T, Strauss G. Strength assessment by isokinetic dynamometry. Physiological Tests for Elite Athletes. 2000:155-99.

14. Van Dyk N BF, Whiteley R. Including the Nordic hamstring exercise in injuryprevention programmes halves the rate of hamstring injuries: a systematic review and meta-analysis of 8459 athletes. British Journal of Sports Medicine. 27. 2019;1136. 
15. Buckthorpe M, Wright S, Bruce-Low S, Nanni G, Sturdy T, Gross AS, et al. Recommendations for hamstring injury prevention in elite football: translating research into practice. British Journal of Sports Medicine. 2019;53(7):449. doi: 10.1136/bjsports-2018099616.

16. Duhig S, Shield AJ, Opar D, Gabbett TJ, Ferguson C, Williams M. Effect of high-speed running on hamstring strain injury risk. British Journal of Sports Medicine. 2016;50(24):1536-40. doi: 10.1136/bjsports-2015-095679.

17. Orchard JW. Hamstrings are most susceptible to injury during the early stance phase of sprinting. British Journal of Sports Medicine: BMJ Publishing Group Ltd and British Association of Sport and Exercise Medicine; 2012. DOI:10.1136/bjsports-2011-090127

18. Fuller CW, Ashton T, Brooks JH, Cancea RJ, Hall J, Kemp SP. Injury risks associated with tackling in rugby union. British Journal of Sports Medicine. 2010;44(3):159-67. doi: 10.1136/bjsm.2008.050864.

19. Williams S, Trewartha G, Kemp SP, Brooks JH, Fuller CW, Taylor AE, et al. Time loss injuries compromise team success in Elite Rugby Union: a 7-year prospective study. British Journal of Sports Medicine. 2016;50(11):651-6. doi: 10.1136/bjsports-2015-094798.

20. Page MJ, McKenzie JE, Bossuyt PM, Boutron I, Hoffmann TC, Mulrow CD, et al. Updating guidance for reporting systematic reviews: development of the PRISMA 2020 statement. Journal of Clinical Epidemiology. 2021;134:103-12. doi: https://doi.org/10.1016/j.jclinepi.2021.02.003.

21. Modesti PA, Reboldi G, Cappuccio FP, Agyemang C, Remuzzi G, Rapi S, et al. Panethnic differences in blood pressure in Europe: a systematic review and meta-analysis. PloS One. 2016;11(1):e0147601. doi: 10.1371/journal.pone.0147601. 
22. Hootman JM, Driban JB, Sitler MR, Harris KP, Cattano NM. Reliability and validity of three quality rating instruments for systematic reviews of observational studies. Research Synthesis Methods. 2011;2(2):110-8. doi: https://doi.org/10.1002/jrsm.41.

23. Hoegberg LCG, Refsgaard F, Pedersen SH, Personne M, Ullah S, Panagiotidis G, et al. Potential pharmacobezoar formation of large size extended-release tablets and their dissolution - an in vitro study. Clinical Toxicology (Phila). 2018:1-11. doi: 10.1080/15563650.2018.1513138.

24. Sterne JAC HM, McAleenan A, Reeves BC, Higgins JPT. Cochrane Handbook for Systematic Reviews of Interventions version 6.2 updated February 2021.

25. Peterson J, Welch V, Losos M, Tugwell P. The Newcastle-Ottawa scale (NOS) for assessing the quality of nonrandomised studies in meta-analyses. Ottawa: Ottawa Hospital Research Institute. 2011.

26. Anastasi SM, Hamzeh MA. Does the eccentric Nordic Hamstring exercise have an effect on isokinetic muscle strength imbalance and dynamic jumping performance in female rugby union players?. Isokinetics and Exercise Science. 2011;19(4):251-60. doi: 10.3233/IES-20110420

27. Reid LC, Cowman JR, Green BS, Coughlan GF. Return to play in elite rugby union: application of global positioning system technology in return-to-running programs. Journal of Sport Rehabilitation. 2013;22(2):122-9. DOI 10.1123/jsr.22.2.122

28. Brown, Brughelli M, Griffiths PC, Cronin JB. Lower-extremity isokinetic strength profiling in professional rugby league and rugby union. International Journal of Sports Physiology and Performance. 2014;9(2):358-61. doi: 10.1123/ijspp.2013-0129. 
29. Deighan MA, Serpell BG, Bitcon MJ, Croix MDS. Knee joint strength ratios and effects of hip position in rugby players. The Journal of Strength \& Conditioning Research. 2012;26(7):1959-66. DOI: 10.1519/JSC.0b013e318234eb46

30. Brown, Brughelli M, Bridgeman LA. Profiling Isokinetic Strength by Leg Preference and Position in Rugby Union Athletes. International Journal of Sports Physiology and Performance. 2016;11(4):500. doi: 10.1123/ijspp.2015-0241.

31. Beyer KS, Fukuda DH, Miramonti AM, Church DD, Tanigawa S, Stout JR, et al. Strength ratios are affected by years of experience in American collegiate rugby athletes: A preliminary study. Isokinetics and Exercise Science. 2016;24(3):257-62. DOI: 10.3233/IES160625

32. Farnan D, Mahony N, Wilson F, Gissane C. A 3-month prospective study of injuries in amateur rugby and soccer. Physiotherapy Practice and Research. 2013;34(2):103-12. DOI: 10.3233/PPR-130023 33. Mendiguchia J, Edouard P, Samozino P, Brughelli M, Cross M, Ross A, et al. Field monitoring of sprinting power-force-velocity profile before, during and after hamstring injury: two case reports. Journal of Sports Science. 2016;34(6):535-41. doi: 10.1080/02640414.2015.1122207.

34. Yamada M, Mastumoto D. The reaction time of mental rotation predicts strain in rugby players. Journal of Physical Therapy Science. 2009;21(2):177-81. doi: https://doi.org/10.1589/jpts.21.177.

35. Upton P, Noakes T, Juritz J. Thermal pants may reduce the risk of recurrent hamstring injuries in rugby players. British Journal of Sports Medicine. 1996;30(1):57-60. doi: 10.1136/bjsm.30.1.57. 
36. Mondin D, Owen JA, Negro M, D'antona G. Validity and reliability of a non-invasive test to assess Quadriceps and Hamstrings strength in athletes. Frontiers in Physiology. 2018;9:1702. doi: https://doi.org/10.3389/fphys.2018.01702.

37. Bourne MN, Opar DA, Williams MD, Shield AJ. Eccentric Knee Flexor Strength and Risk of Hamstring Injuries in Rugby Union: A Prospective Study. The American Journal of Sports Medicine. 2015;43(11):2663-70. doi: 10.1177/0363546515599633.

38. Brooks JH, Fuller CW, Kemp SP, Reddin DB. Epidemiology of injuries in English professional rugby union: part 1 match injuries. British Journal of Sports Medicine. 2005;39(10):757-66. doi: 10.1136/bjsm.2005.018135.

39. Brooks JH, Fuller CW, Kemp SP, Reddin DB. Epidemiology of injuries in English professional rugby union: part 2 training Injuries. British Journal of Sports Medicine. 2005;39(10):767-75. doi: 10.1136/bjsm.2005.018408.

40. Turl SE, George KP. Adverse Neural Tension: A Factor in Repetitive Hamstring Strain? Journal of Orthopaedic \& Sports Physical Therapy. 1998;27(1):16-21. doi: 10.2519/jospt.1998.27.1.16.

41. Severo-Silveira L, Dornelles MP, Lima-e-Silva FX, Marchiori CL, Medeiros TM, Pappas E, et al. Progressive Workload Periodization Maximizes Effects of Nordic Hamstring Exercise on Muscle Injury Risk Factors. Journal of Strength and Conditioning Research. 2018. doi: 10.1519/JSC.0000000000002849.

42. Roberts SP, Trewartha G, England M, Shaddick G, Stokes KA. Epidemiology of timeloss injuries in English community-level rugby union. British Medicine Journal 2013;3(11):e003998. doi: http://dx.doi.org/10.1136/bmjopen-2013-003998. 
43. Brooks JH, Kemp SP. Injury-prevention priorities according to playing position in professional rugby union players. British Journal of Sports Medicine. 2011;45(10):765-75. doi: 10.1136/bjsm.2009.066985.

44. Kenneally-Dabrowski C, Brown NA, Warmenhoven J, Serpell BG, Perriman D, Lai AK, et al. Late swing running mechanics influence hamstring injury susceptibility in elite rugby athletes: A prospective exploratory analysis. Journal of Biomechanics. 2019. DOI https://doi.org/10.1016/j.jbiomech.2019.05.037

45. Kenneally-Dabrowski C, Serpell BG, Spratford W, Lai AK, Field B, Brown NA, et al. A retrospective analysis of hamstring injuries in elite rugby athletes: More severe injuries are likely to occur at the distal myofascial junction. Physical Therapy in Sport. 2019. DOI https://doi.org/10.1016/j.ptsp.2019.05.009

46. Dobbs IJ, Watkins CM, Barillas SR, Wong MA, Brown LE. Assessing knee strength ratios and bilateral deficit via dynamic vs. static tests in amateur rugby union players. Isokinetics and Exercise Science. 2017;25(4):281-7. DOI: 10.3233/IES-17117147. Abdelfettah Y, Ngandzali DD, Ouazzani H, Erouam B, El Hassan K. Isokinetic Assessment of the Knee of Amateur Soccer and Amateur Rugby Players. Critical Reviews ${ }^{\mathrm{TM}}$ in Physical and Rehabilitation $\quad$ Medicine. 2019;31(4). doi: 10.1615/CritRevPhysRehabilMed.2020032808.

48. Yeomans C, Comyns TM, Cahalan R, Hayes K, Costello V, Warrington GD, et al. The relationship between physical and wellness measures and injury in amateur rugby union players. Physical Therapy in Sport. 2019;40:59-65. doi: 10.1016/j.ptsp.2019.08.012.

49. Berry JG, Harrison JE, Yeo JD, Cripps RA, Stephenson SC. Cervical spinal cord injury in rugby union and rugby league: are incidence rates declining in NSW? Australian and New 
Zealand Journal of Public Health.. 2006;30(3):268-74. DOI: $10.1177 / 03635465980260021201$

50. Harding AT, Weeks BK, Horan SA, Little A, Watson SL, Beck BR. Validity and testretest reliability of a novel simple back extensor muscle strength test. SAGE Open Medicine. 2017;5:2050312116688842. doi: 2050312116688842.

51. Aagaard P, Simonsen EB, Magnusson SP, Larsson B, Dyhre-Poulsen P. A new concept for isokinetic hamstring: quadriceps muscle strength ratio. The American Journal of Sports Medicine. 1998;26(2):231-7. DOI:10.1177/03635465980260021201.

52. Bourne MN, Opar DA, Williams MD, Shield AJ. Eccentric Knee Flexor Strength and Risk of Hamstring Injuries in Rugby Union: A Prospective Study. American Journal of Sports Medicine. 2015;43(11):2663-70. doi: 10.1177/0363546515599633.

53. Van Dyk N, Wangensteen A, Vermeulen R, Whiteley R, Bahr R, Tol J, et al. Similar Isokinetic Strength Preinjury and at Return to Sport after Hamstring Injury. Medicne Science and Sports Exercise 2019. doi: 10.1249/MSS.0000000000001900.

54. Malone S, Roe M, Doran DA, Gabbett TJ, Collins K. High chronic training loads and exposure to bouts of maximal velocity running reduce injury risk in elite Gaelic football. Journal of science and medicine in sport. 2017;20(3):250-4.

55. Crema MD, Guermazi A, Tol JL, Niu J, Hamilton B, Roemer FW. Acute hamstring injury in football players: association between anatomical location and extent of injury-A large single-center MRI report. Journal of Science and Medicine in Sport. 2016;19(4):317-22. doi: https://doi.org/10.1016/j.jsams.2015.04.005.

56. Hegyi A, Péter A, Finni T, Cronin N. Region dependent hamstrings activity in Nordic hamstring exercise and stiff leg deadlift defined with high density electromyography. 
Scandinavian Journal of Medicine \& Science in Sports. 2018;28(3):992-1000. DOI:10.1177/03635465980260021201.

57. Smart D, Hopkins W, Gill N. Differences and Changes in the Physical Characteristics of Professional and Amateur Rugby Union Players. Journal of Strength and Conditioning Research. 2013;27(11):3033-44. doi: 10.1519/JSC.0b013e31828c26d3.

59. Coombs R, Garbutt G. Developments in the use of the hamstring/quadriceps ratio for the assessment of muscle balance. Journal of Sports Science \& Medicine. 2002;1(3):56. . PMCID: PMC3967430

60. Van Dyk N, Bahr R, Burnett AF, Whiteley R, Bakken A, Mosler A, et al. A comprehensive strength testing protocol offers no clinical value in predicting risk of hamstring injury: a prospective cohort study of 413 professional football players. British Journal of Sports Medicine. 2017;51(23):1695-702. doi: 10.1136/bjsports-2017-097754.

61. Hewett TE, Myer GD, Zazulak BT. Hamstrings to quadriceps peak torque ratios diverge between sexes with increasing isokinetic angular velocity. Journal of Science and Medicine in Sport. 2008;11(5):452-9.

62. Hewett TE, Myer GD, Ford KR. Decrease in neuromuscular control about the knee with maturation in female athletes. The Journal of Bone and Joint Surgery. 2004;86(8):1601-8. doi: 10.2106/00004623-200408000-00001.

63. Kyrolainen H, Komi PV, Belli A. Changes in muscle activity patterns and kinetics with increasing running speed. The Journal of Strength \& Conditioning Research. 1999;13(4):4006. doi: $10.1080 / 02640410400021575$ 
64. Kalkhoven JT, Watsford ML, McLean BD, Sides DL. The evidence indicates that the hamstrings do not behave isometrically during the swing phase of the sprint cycle: a narrative review. SportRxiv. 2020. doi: 10.31236/osf.io/8ujt2.

65. Claudino JG, Cardoso Filho CA, Bittencourt NFN, Gonçalves LG, Couto CR, Quintão RC, et al. Eccentric Strength Assessment of Hamstring Muscles with New Technologies: a Systematic Review of Current Methods and Clinical Implications. Sports Medicine-Open. 2021;7(1):1-15. doi: https://doi.org/10.1186/s40798-021-00298-7.

66. Timmins RG, Bourne MN, Shield AJ, Williams MD, Lorenzen C, Opar DA. Short biceps femoris fascicles and eccentric knee flexor weakness increase the risk of hamstring injury in elite football (soccer): a prospective cohort study. British Journal of Sports Medicine. 2016;50(24):1524-35. doi: 10.1136/bjsports-2015-095362.

67. Giakoumis. To Nordic or not to Nordic?A different perspective with reason to appreciate Semitendinosus more than ever. Sport Perfomance and Science Reports. 2020.

\section{Figures legends}

Figure 1. Preferred Reporting Items for Systematic Reviews and Meta-Analyses flow chart.

Figure 2. Illustration of a Nordic hamstring exercise. 


\section{Tables}

Table 1. Characteristics of the 24 included studies.

Study

Abdelfettah

Anastasi, Hamzeh Moderate

5 stars

\section{NOS rating* \\ Player characteristics}

10 male amateur players

Age: $26.4 \pm 5.1 \mathrm{y}$

Mass: $83.2 \pm 15.3 \mathrm{~kg}$

Height: $177.9 \pm 8.3 \mathrm{~cm}$

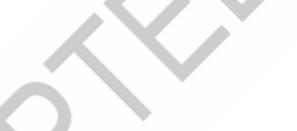

24 female amateur players (13 Case control test players and 11 controls)

Study design

Location

Cohort

Morocco (cross-sectional)

Age: $25.2 \pm 5.3 \mathrm{y}$

Mass: $69.5 \pm 10.9 \mathrm{~kg}$

Height: $166 \pm 5.0 \mathrm{~cm}$

\section{Key study}

characteristics

Compare the strength of the Duration: 1 season

knee flexors and extensors Injuries: No

between soccer and rugby Intervention: No

players and the level of

strength by playing positions

Evaluate the effect of Duration: 1 season

hamstring eccentric program Injuries: No

on leg strength imbalance and Intervention: Yes

maximal vertical jump height 


\begin{tabular}{|c|c|c|c|c|c|}
\hline \multirow[t]{4}{*}{ Beyer et al. [31] } & Moderate & 25 male amateur players. & series & Compare isotonic versus & Duration: 1 season \\
\hline & 4 stars & Age: $20.7 \pm 2 y$ & (laboratory & isometric strength measures & Injuries: No \\
\hline & & Mass: $86.8 \pm 15.4 \mathrm{~kg}$ & investigation) & between players with $<2$ & Intervention: No \\
\hline & & Height: $179 \pm 8.0 \mathrm{~cm}$ & USA & years and $\geq 2$ years of & \\
\hline \multirow[t]{5}{*}{ Bourne et al. [37] } & Strong & 178 male players & Cohort & Determine the thresholds of & Duration: 1 season \\
\hline & 8 stars & professionals and 103 semi- & (prospective) & hamstring strength imbalance & Injuries: Yes \\
\hline & & professionals subdivided in 65 & Australia & in eccentric Nordic exercises & Intervention: No \\
\hline & & sub-elite and 38 under & & association with & \\
\hline & & Age: $22.6 \pm 3$ & & hamstring injuries & \\
\hline \multirow[t]{4}{*}{ Brooks et al. [38] } & Strong & 546 male professional players & Cohort & Study match injuries in & Duration: \\
\hline & 9 stars & Age: $25.3 \pm 4.1 \mathrm{y}$ & (prospective) & players and & seasons \\
\hline & & Mass: $100.0 \pm 12.1 \mathrm{~kg}$ & United Kingdom & define incidence, aetiology, & Injuries: Yes \\
\hline & & Height: $185.1 \pm 7.4 \mathrm{~cm}$ & & and severity & Intervention: No \\
\hline
\end{tabular}




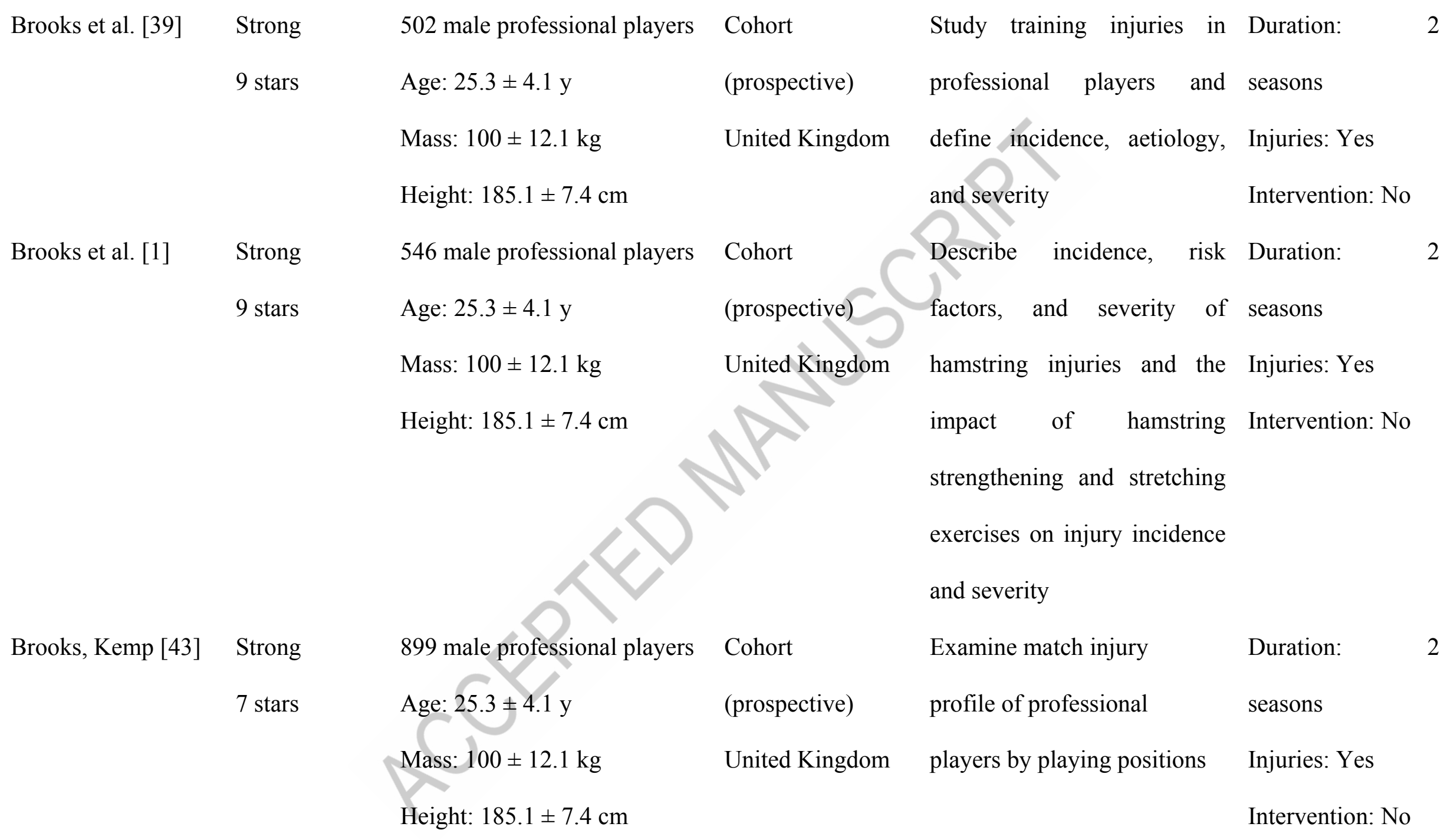




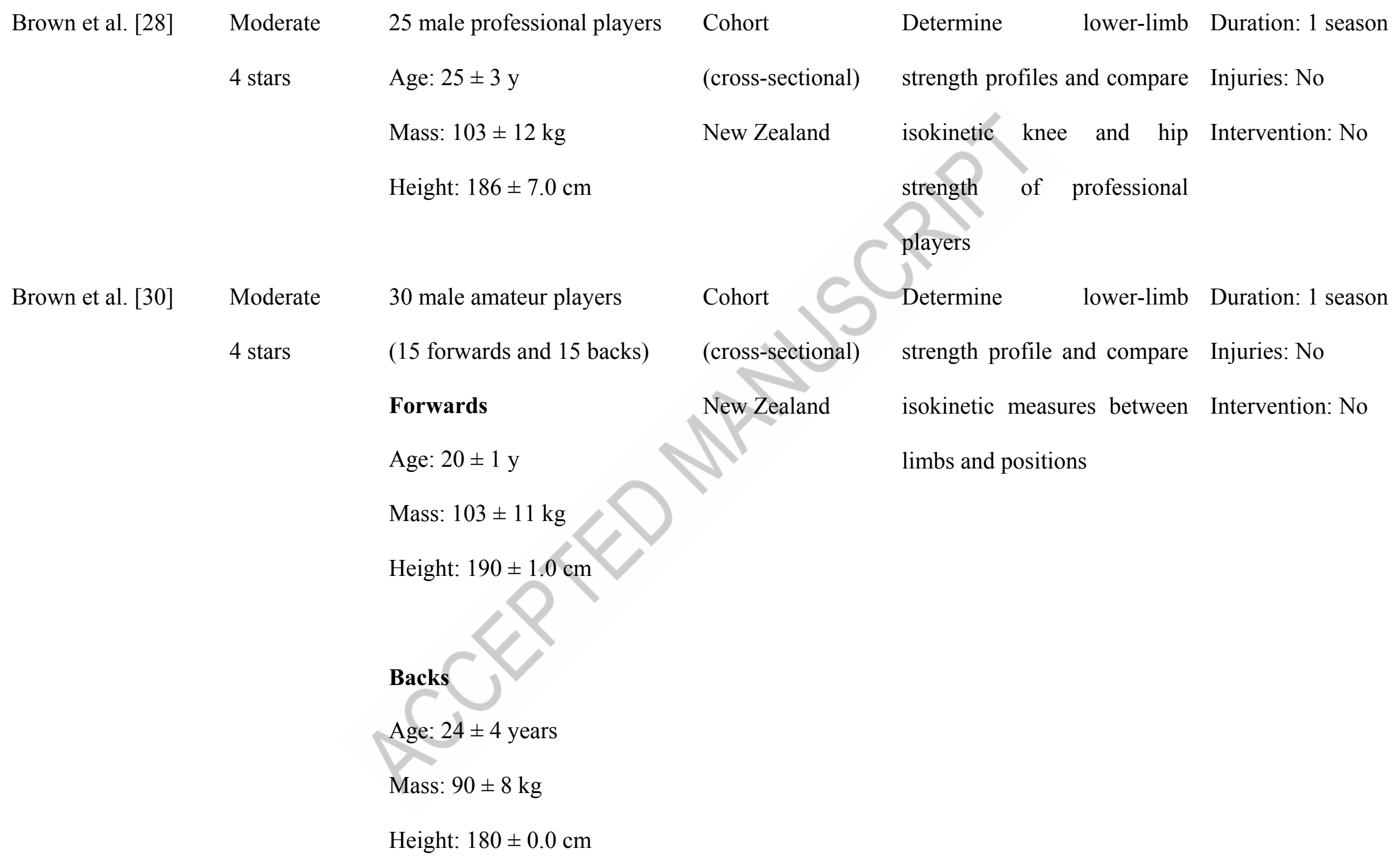




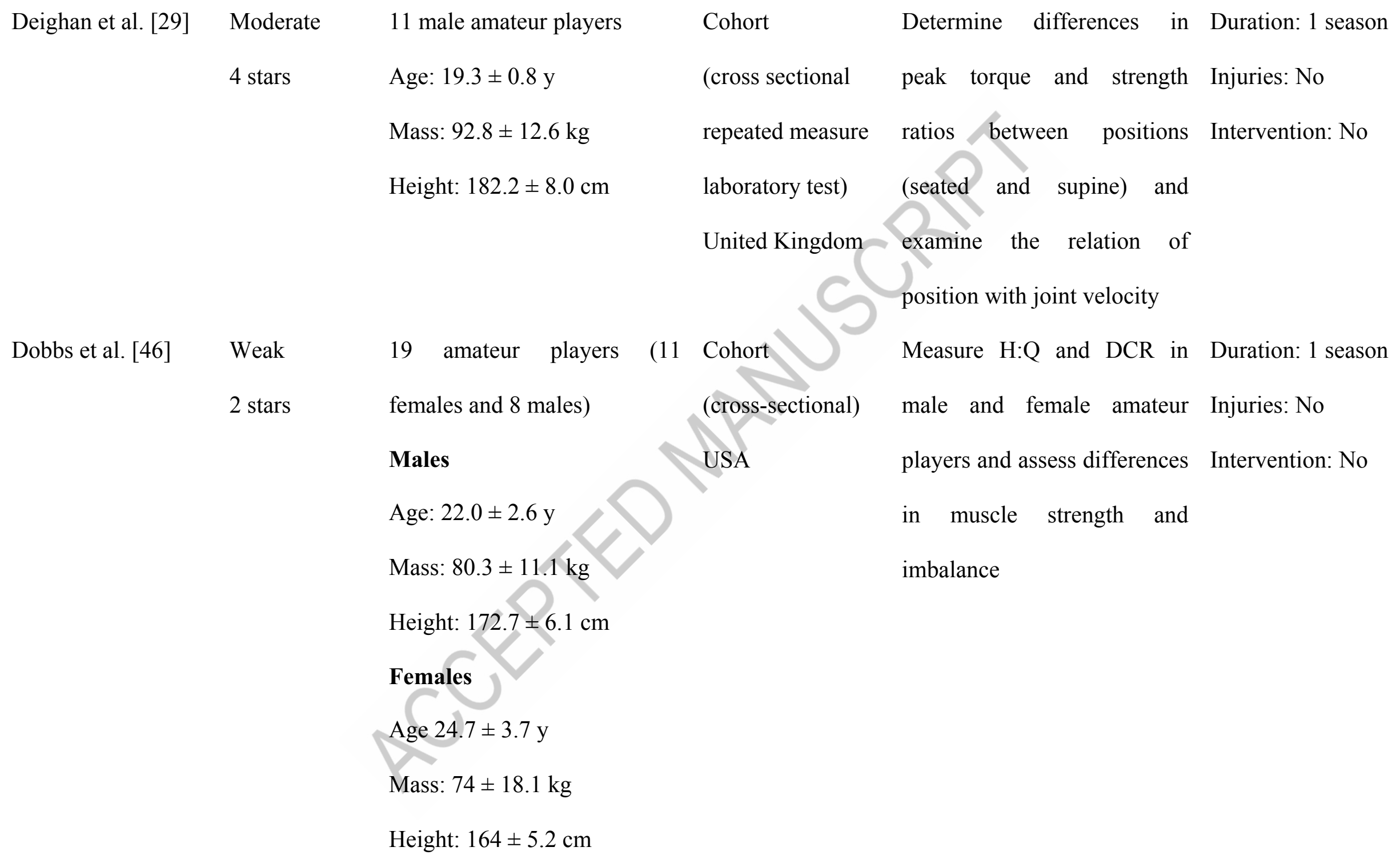




\begin{tabular}{|c|c|c|c|c|c|}
\hline Farnan et al. [32] & Moderate & 54 male amateur players & Cohort & Determine hamstring injuries & Duration: 1 season \\
\hline & 5 stars & Age: $21 \pm 2 y$ & (prospective) & incidence, and severity & Injuries: Yes \\
\hline & & Mass: $88.1 \pm 10.7 \mathrm{~kg}$ & Ireland & & Intervention: No \\
\hline & & Height: $183 \pm 5.2 \mathrm{~cm}$ & & & \\
\hline Kenneally- & Strong & 74 male professional players & Cohort & hamstring injuries & Duration: \\
\hline Dabrowski et al. & 7 stars & Age: NR & (retr & (severity, grade, and location) & seasons \\
\hline [45] & & Mass: NR & & and the relationship with & Injuries: Yes \\
\hline & & Height: NR & & game demands & Intervention: No \\
\hline Kenneally- & Strong & 10 male professional $\mathrm{r}$ & Cohort & Analysis of the relationship & Duration: 1 season \\
\hline Dabrowski et al. & 7 stars & Age: $27.3 \pm 3.2 \mathrm{y}$ & (prospective) & between overground high- & Injuries: Yes \\
\hline [44] & & Mass: 100. & Australia & speed running mechanics and & Intervention: No \\
\hline & & Height :193 & & hamstring injury & \\
\hline Mendiguchia et al. & Moderate & 1 male professional player & Case series & Determine the changes in & Duration: 1 season \\
\hline [33] & 4 stars & Age: 23 y & (laboratory & sprinting mechanics in & Injuries: No \\
\hline & & Mass: $94 \mathrm{~kg}$ & investigation) & relation to hamstring injuries & Intervention: No \\
\hline & & Height: $187 \mathrm{~cm}$ & New Zeal & & \\
\hline
\end{tabular}




\begin{tabular}{|c|c|c|c|c|c|}
\hline \multirow[t]{5}{*}{ Mondin et al. [36] } & Moderate & 10 male professional players & Cohort & Measure hamstring strength & Duration: 1 season \\
\hline & 4 stars & and 14 healthy controls & (repeated- & with a sphygmomanometer & Injuries: No \\
\hline & & Age: $23.1 \pm 2.5 \mathrm{y}$ & measures & test and the correspondence & Intervention: No \\
\hline & & Mass: $88.4 \pm 8.5 \mathrm{~kg}$ & reliability) & isokinetic & \\
\hline & & Height: $180.9 \pm 8.2 \mathrm{~cm}$ & United Kingdo & dynamometry test. & \\
\hline \multirow[t]{4}{*}{ Reid et al. [27] } & Moderate & 8 male professional players & Case series & Demonstrate the application & Duration: 1 season \\
\hline & 6 stars & Age: $27.9 \pm 4.8 \mathrm{y}$ & Ireland & of GPS & Injuries: No \\
\hline & & Mass: $99.1 \pm 9.9 \mathrm{~kg}$ & & technology & Intervention: No \\
\hline & & Height: 185 & & management of return to play & \\
\hline \multirow[t]{6}{*}{ Roberts et al. [42] } & Strong & 189 male community players & Cohort & Establish injury incidence & Duration: \\
\hline & 7 stars & Group A: semi-professional & (prospective) & and severity in community & seasons \\
\hline & & Group B: amateur & United Kingdom & rugby and assess differences & Injuries: Yes \\
\hline & & Group C: recreational & & between levels & Intervention: No \\
\hline & & Age: NR & & & \\
\hline & & Mass: NR & & & \\
\hline
\end{tabular}


Height: NR

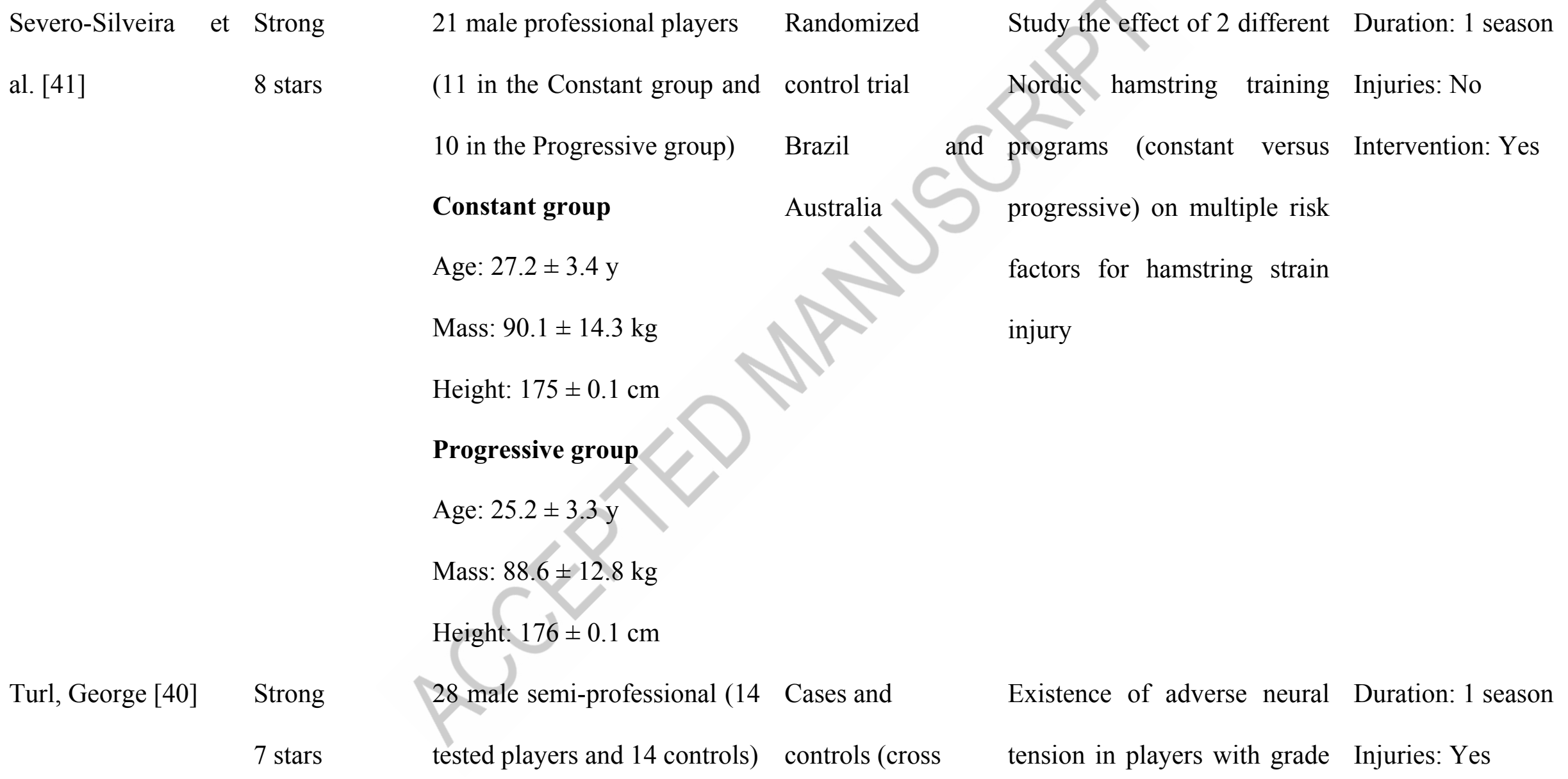


Age: $29 \pm 3$ y

Weight: $88 \pm 12 \mathrm{~kg}$

Height: $181 \pm 1.0 \mathrm{~cm}$

\begin{tabular}{|c|c|c|c|c|c|}
\hline Upton et al. [35] & $\begin{array}{l}\text { Moderate } \\
5 \text { stars }\end{array}$ & $\begin{array}{l}44 \text { male NR level players } \\
\text { Age: } 23 \pm 3 \text { y }\end{array}$ & $\begin{array}{l}\text { Cohort } \\
\text { (prospective) }\end{array}$ & $\begin{array}{l}\text { Determine if the use of } \\
\text { thermal pants reduce the risk }\end{array}$ & $\begin{array}{l}\text { Duration: } 1 \text { season } \\
\text { Injuries: Yes }\end{array}$ \\
\hline & & $\begin{array}{l}\text { Weight: NR } \\
\text { Height: NR }\end{array}$ & South & of hamstring injuries. & Intervention: Yes \\
\hline Yamada, & Moderate & 21 male amateur players & Cohort & Determine the relationship & Duration: 1 season \\
\hline Mastumoto [34] & 5 stars & $\begin{array}{l}\text { Age: } 21.3 \pm 0.3 \mathrm{y} \\
\text { Weight: } 71.8 \pm 6.3 \mathrm{~kg} \\
\text { Height: } 172.2 \pm 4.1 \mathrm{~cm}\end{array}$ & $\begin{array}{l}\text { (laboratory } \\
\text { investigation) } \\
\text { Japan }\end{array}$ & $\begin{array}{l}\text { between motor imagery and } \\
\text { hamstring injuries. }\end{array}$ & $\begin{array}{l}\text { Injuries: Yes } \\
\text { Intervention: No }\end{array}$ \\
\hline Yeomans et al. [48] & $\begin{array}{l}\text { Moderate } \\
6 \text { stars }\end{array}$ & $\begin{array}{l}137 \text { amateur players ( } 13 \text { males } \\
\text { and } 24 \text { females) } \\
\text { Males } \\
\text { Age: } 22.7 \pm 3.9 y\end{array}$ & $\begin{array}{l}\text { Cohort } \\
\text { (prospective) } \\
\text { Ireland }\end{array}$ & $\begin{array}{l}\text { Determine risk factors } \\
\text { associate to injuries. }\end{array}$ & $\begin{array}{l}\text { Duration: } 1 \text { season } \\
\text { Injuries: Yes } \\
\text { Intervention: No }\end{array}$ \\
\hline
\end{tabular}


10.9; Backs $85.4 \pm 7.9 \mathrm{~kg}$

Height: Forwards $180.5 \pm$

23.6; Backs $179.5 \pm 4.8 \mathrm{~cm}$

\section{Females}

Age: $25.6 \pm 4.9$ y

Weight: Forwards $87.3 \pm$

14.1; Backs $69.5 \pm 11.3 \mathrm{~kg}$

Height: Forwards $169.8 \pm 3.8$;

Backs $165.9 \pm 7.1 \mathrm{~cm}$

Notes. Values are means \pm standard deviations.

Abbreviations: DCR, isokinetic dynamic control ratio; $\mathrm{H}: \mathrm{Q}$, isokinetic hamstring quadriceps ratio; GPS, global positioning system; NOS, Newcastle-Ottawa Scale; NR, not reported.

* Weak: 0 to 3 stars; moderate: 4 to 6 stars; strong: 7 to 10 stars. 
Table 2. Hamstring injury studies involving Rugby Union players.

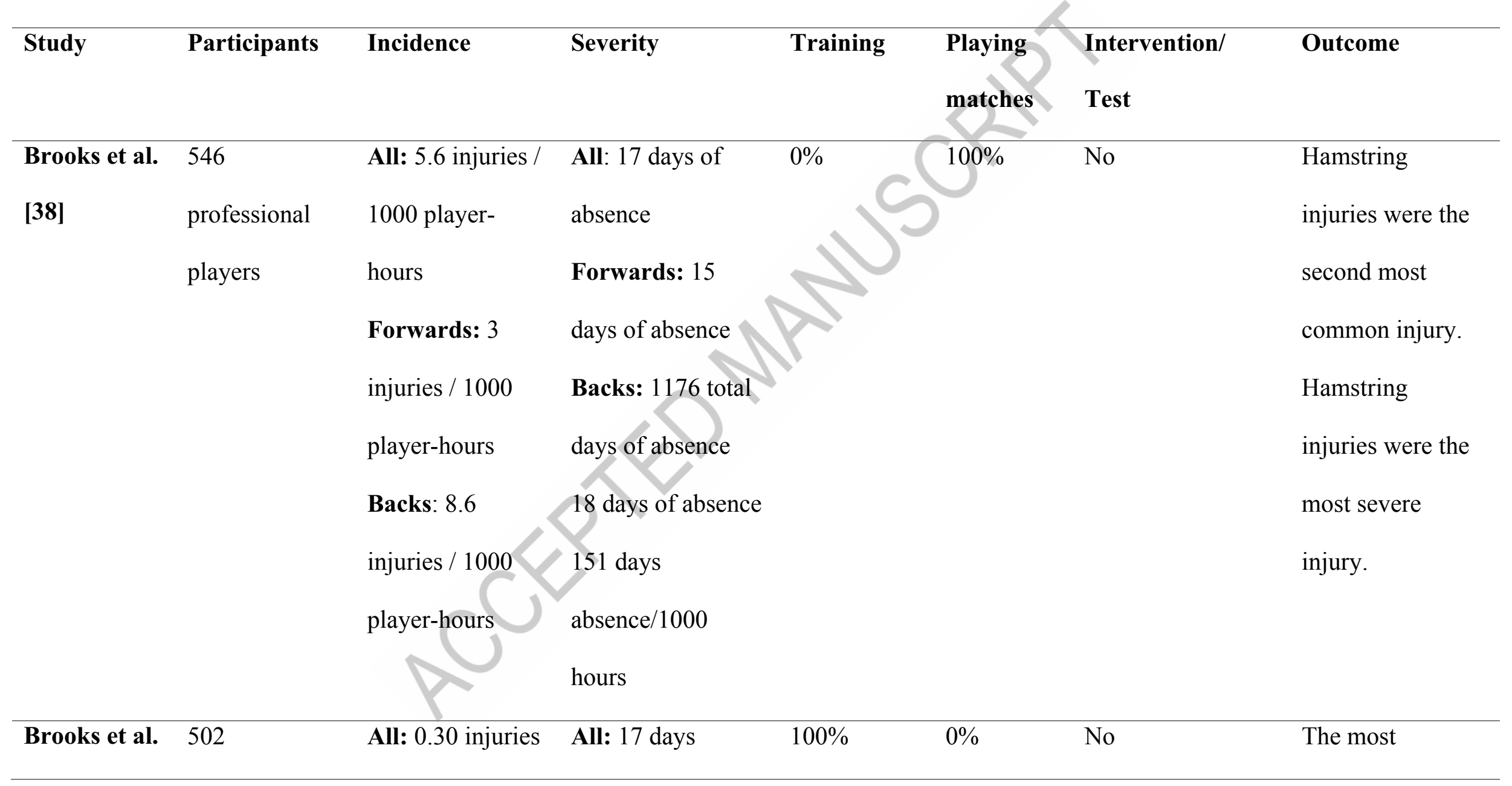




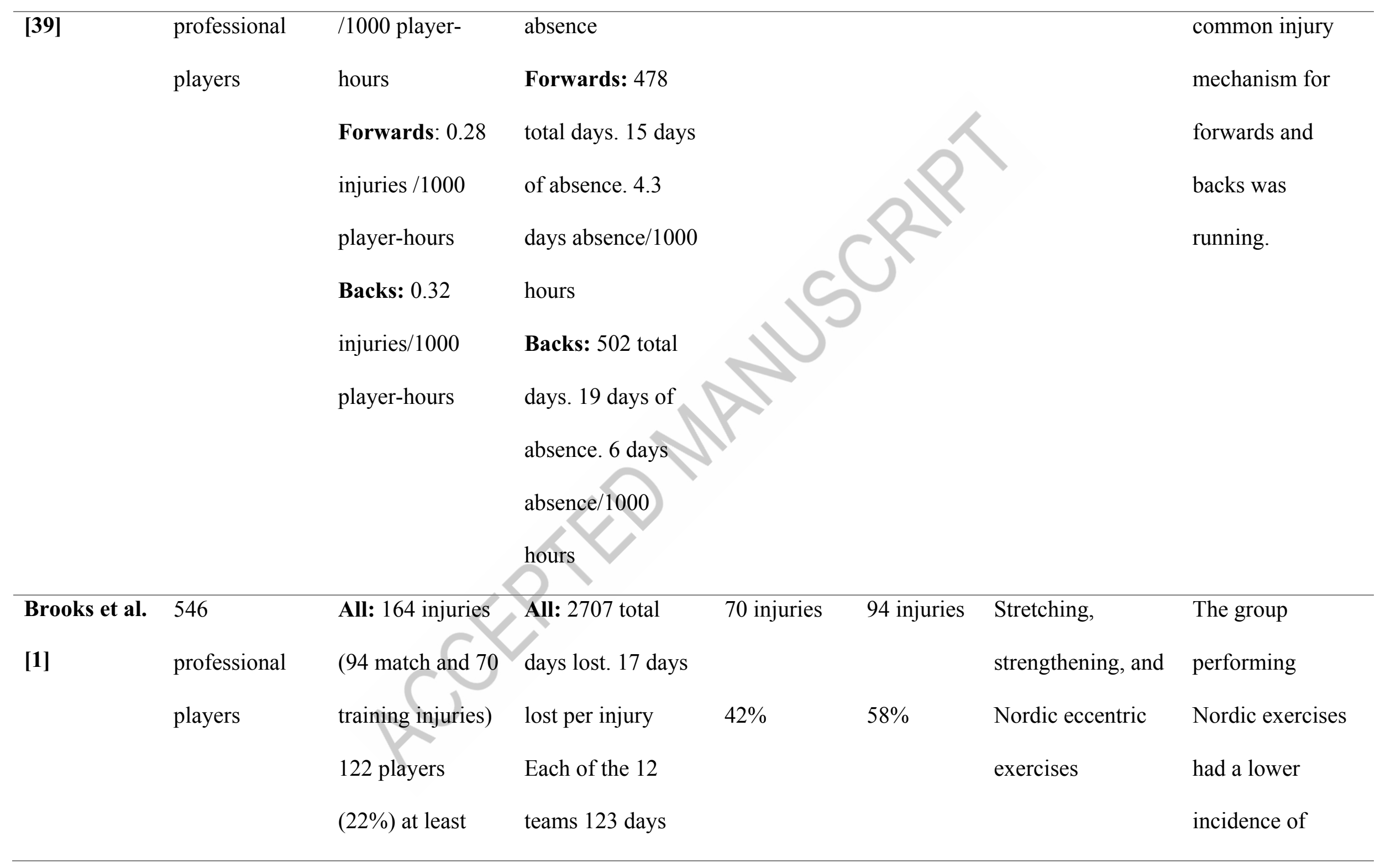




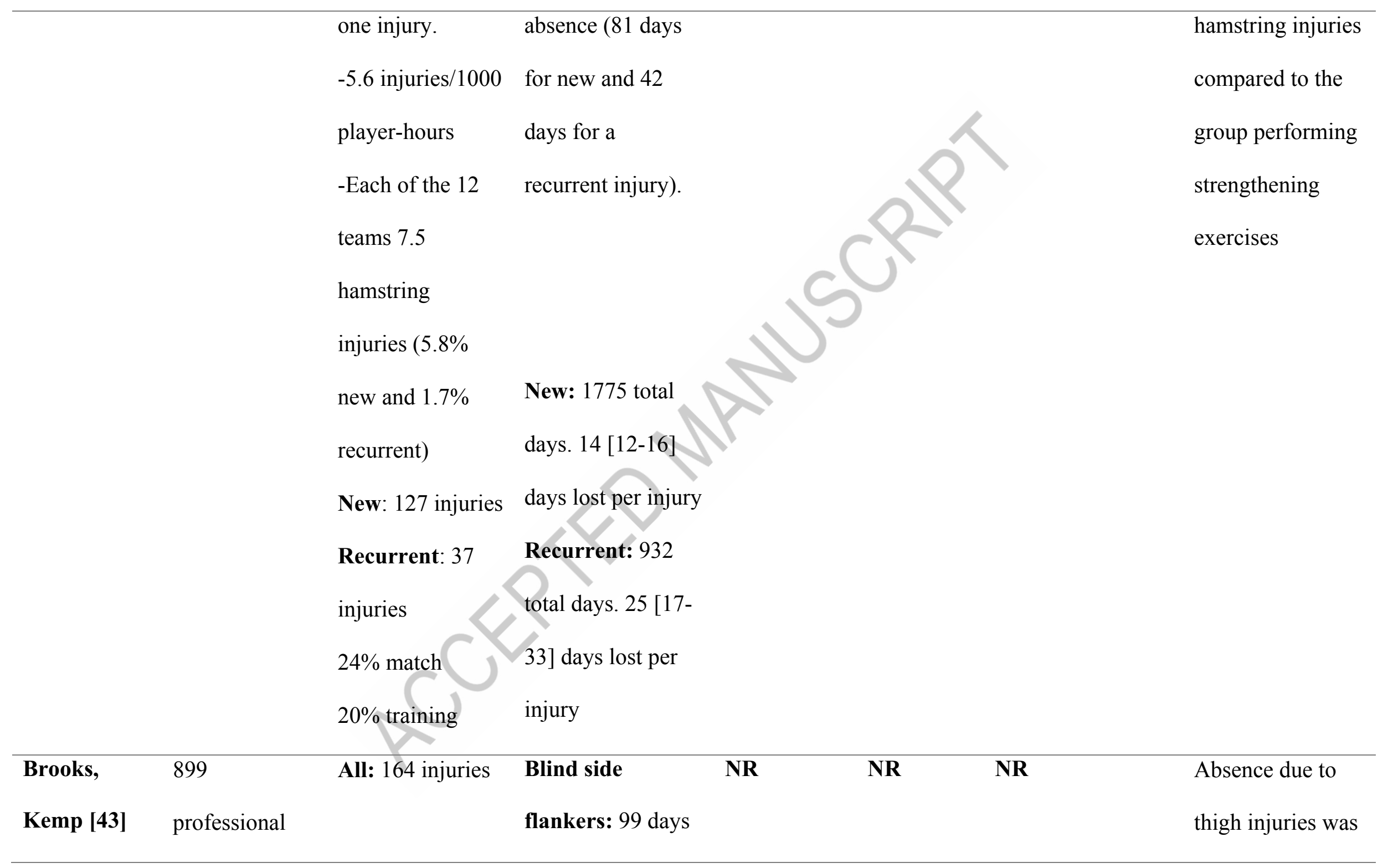




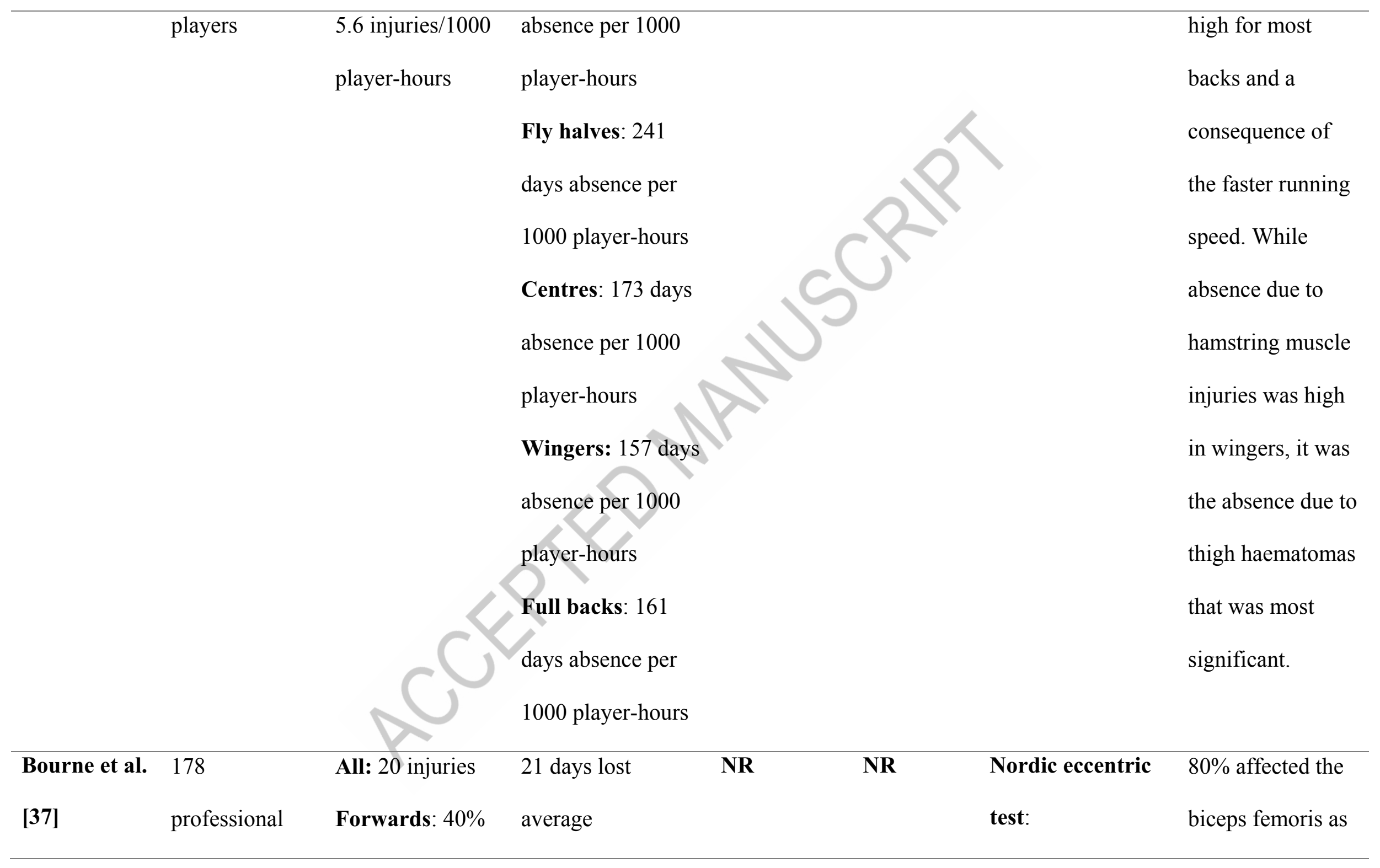




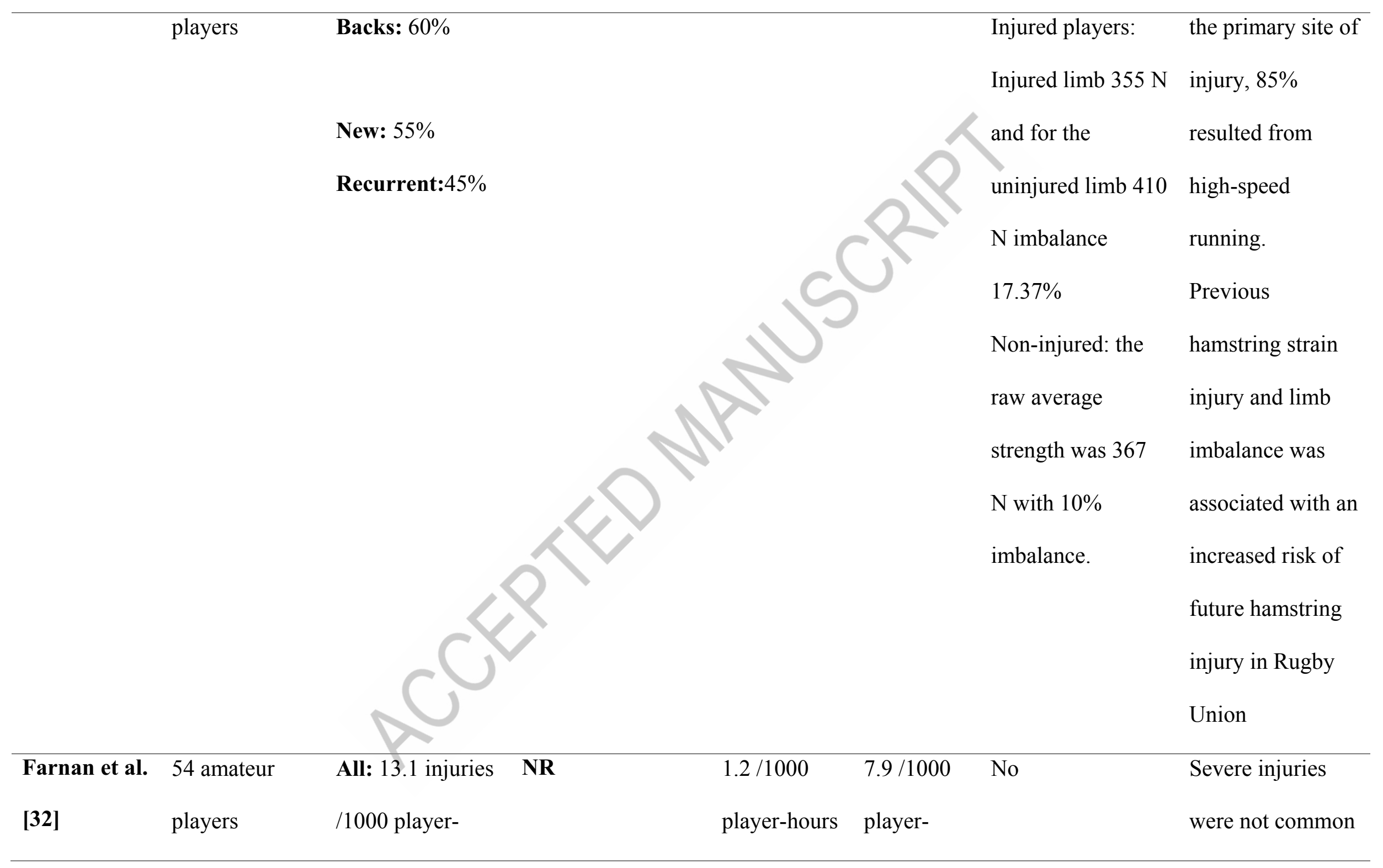




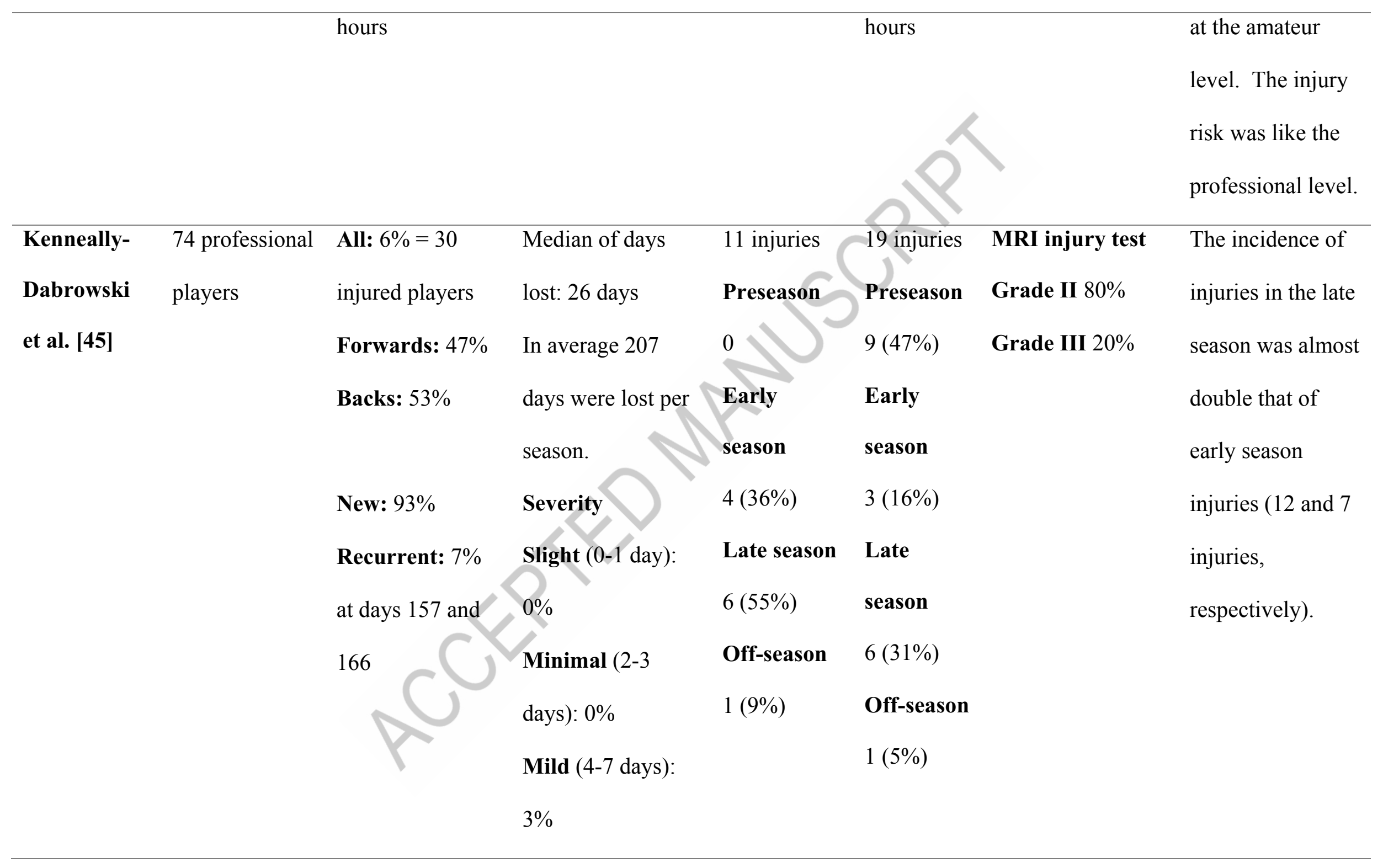




\section{Moderate (7-28}

days): $68 \%$

Severe (more than

28 days): $37 \%$

\section{Career ending:}

$0 \%$

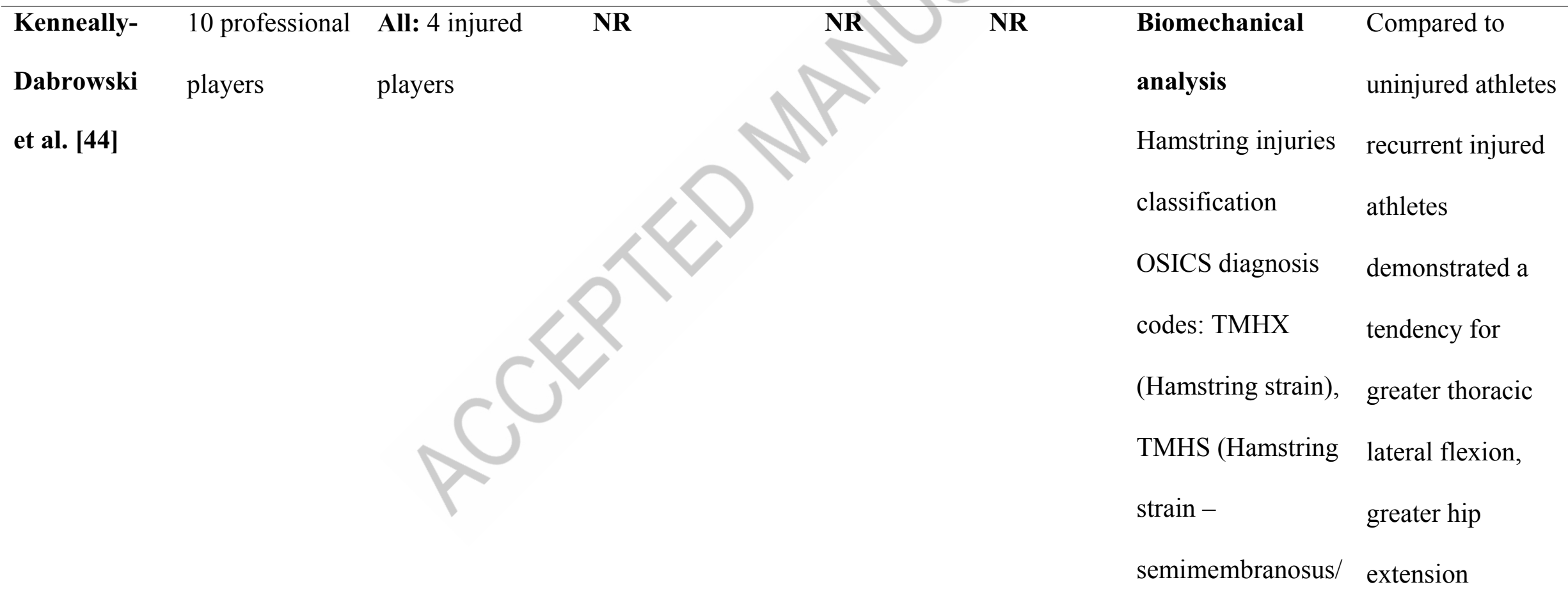


strain, grade 1-2)

and TMHR (Grade

3 hamstring

strain).

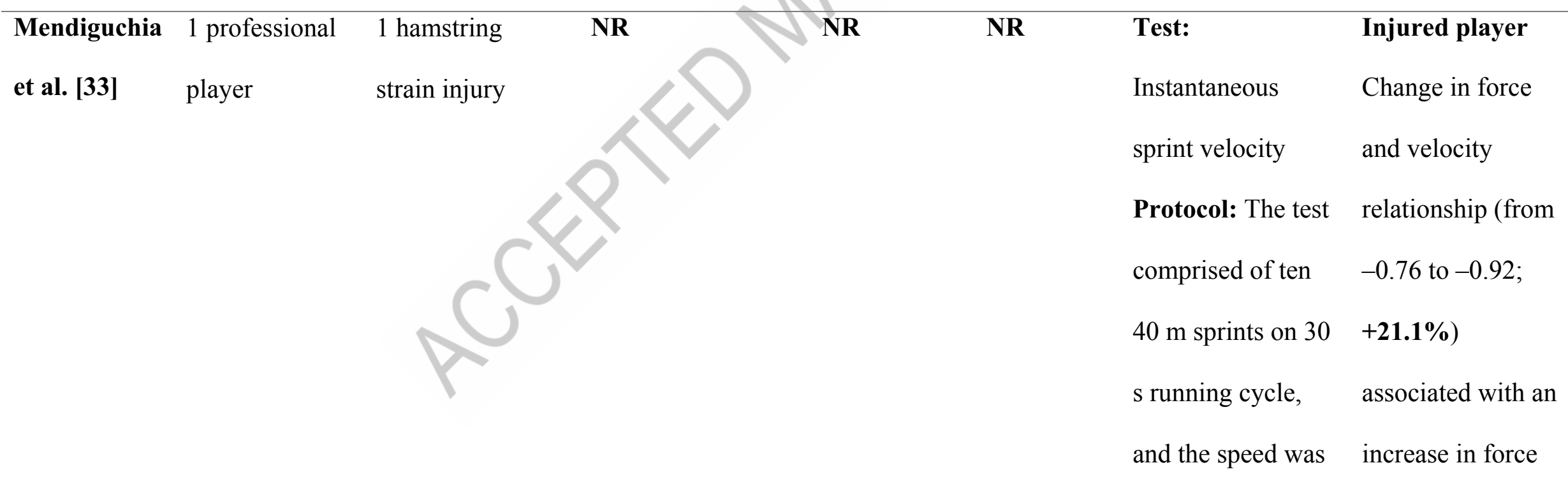




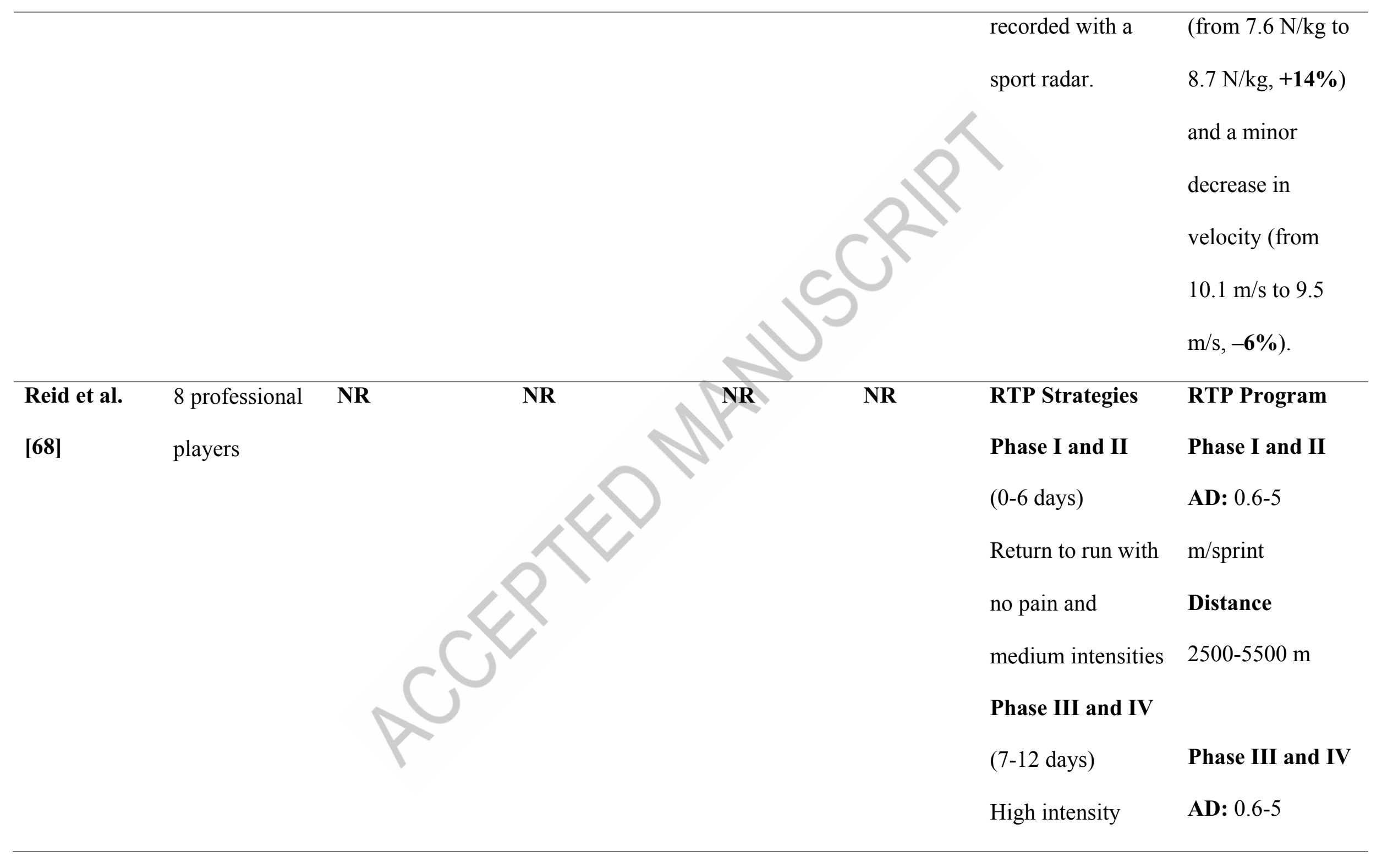




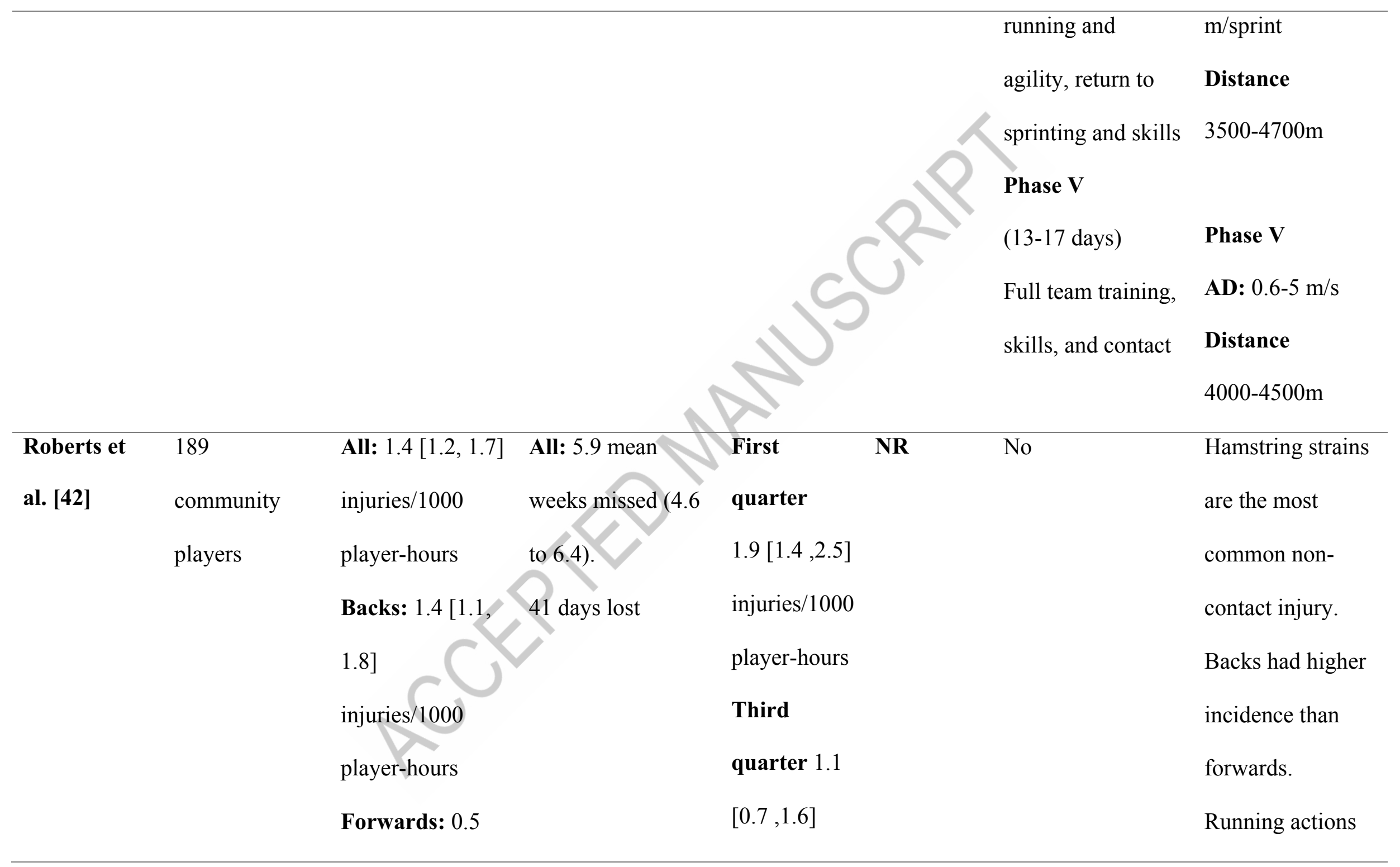


$[0.3,0.7]$

injuries/1000

player-hours

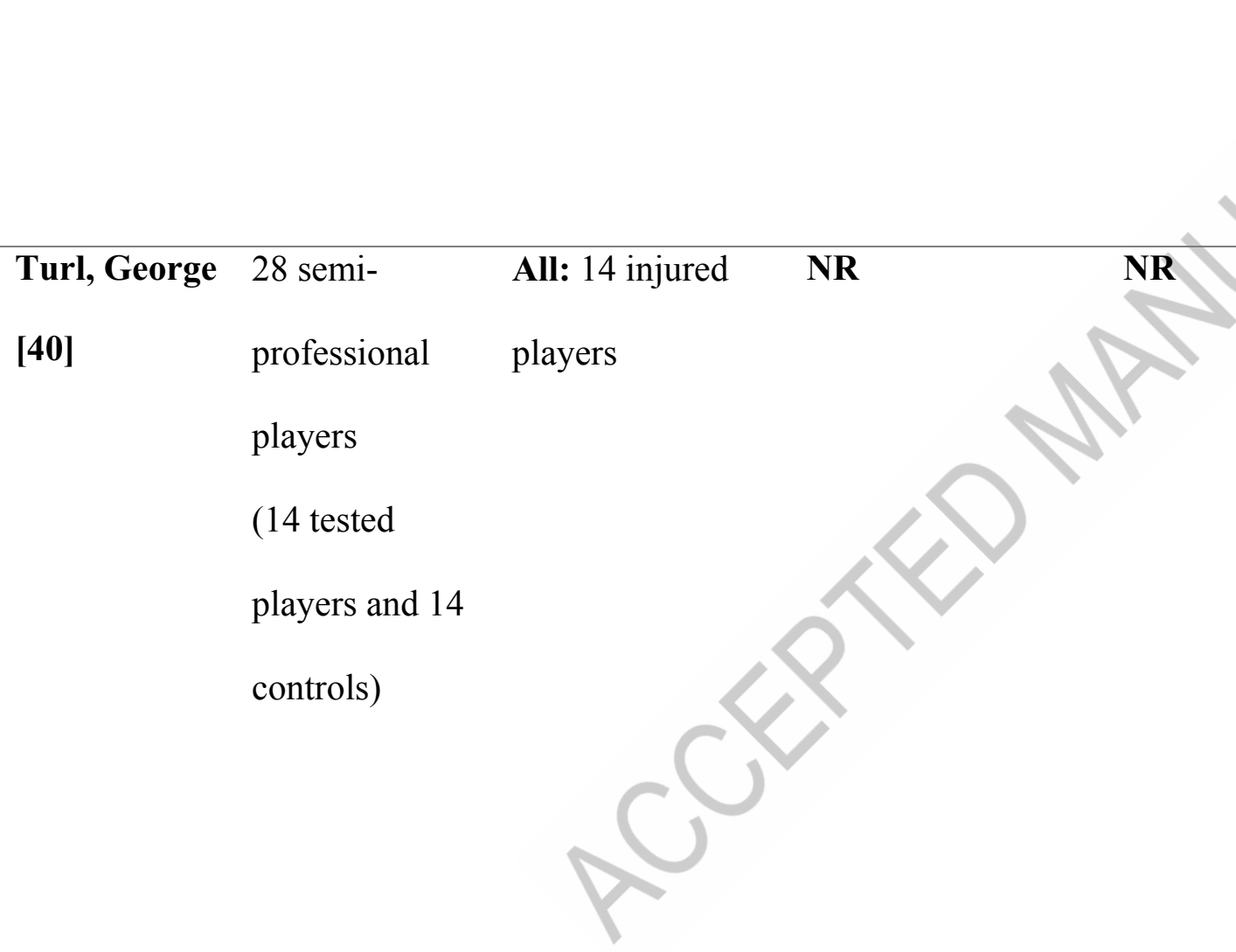

injuries/1000

player-hours

$0 \%$ of all

injuries and of

those $54 \%$ were

hamstring strain

injuries

Slump test: In a

A positive slump

seated position,

test reproduced

with the knees and

radicular

hips at 90 degrees,

symptoms $57 \%$

the player had to

positive in test

slump forward

group, $0 \%$ in

with the chin

control group.

flexed to the chest.

Pressure is applied

to the thoracic 
spine while

extending the

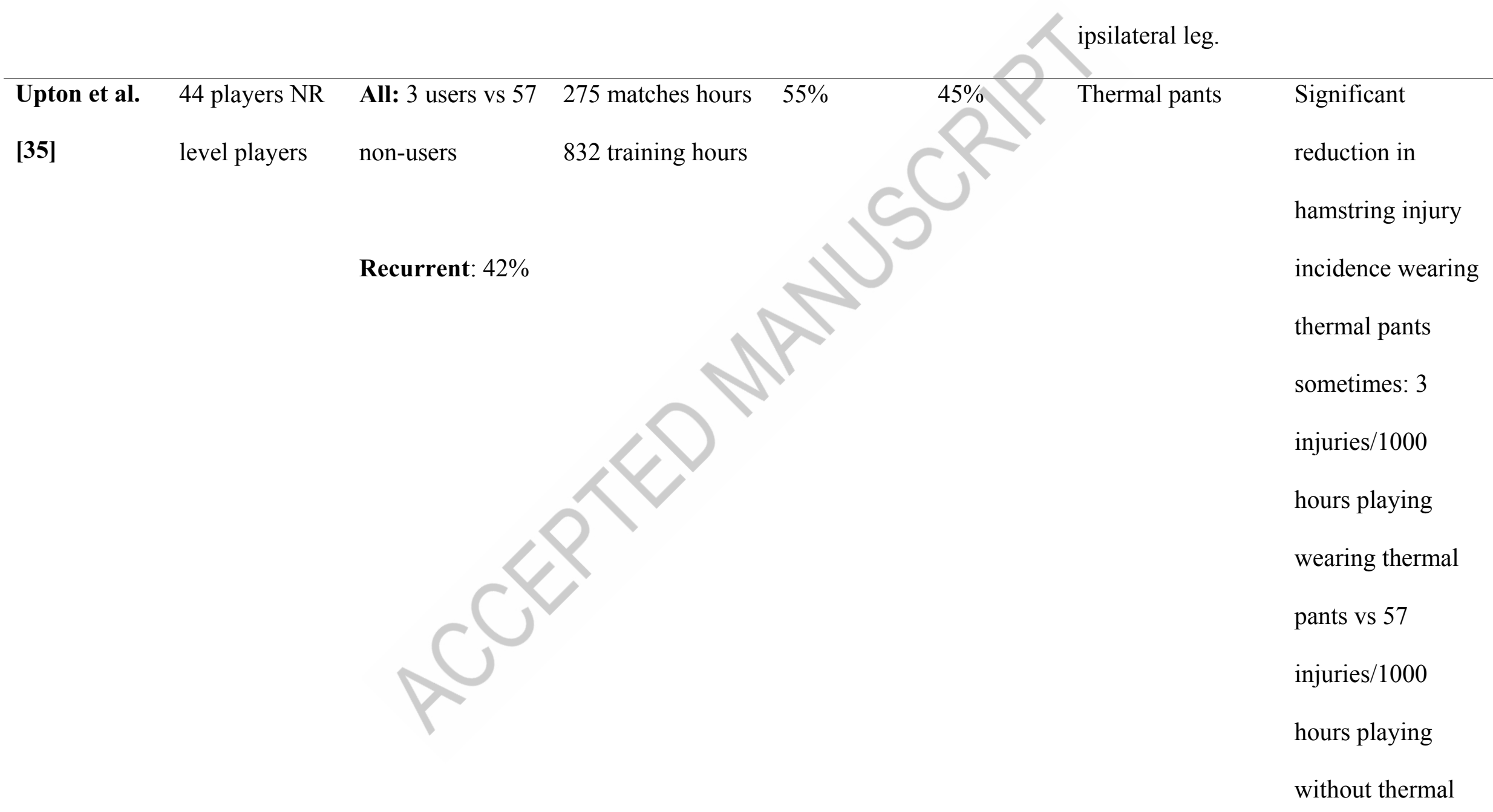


pants.

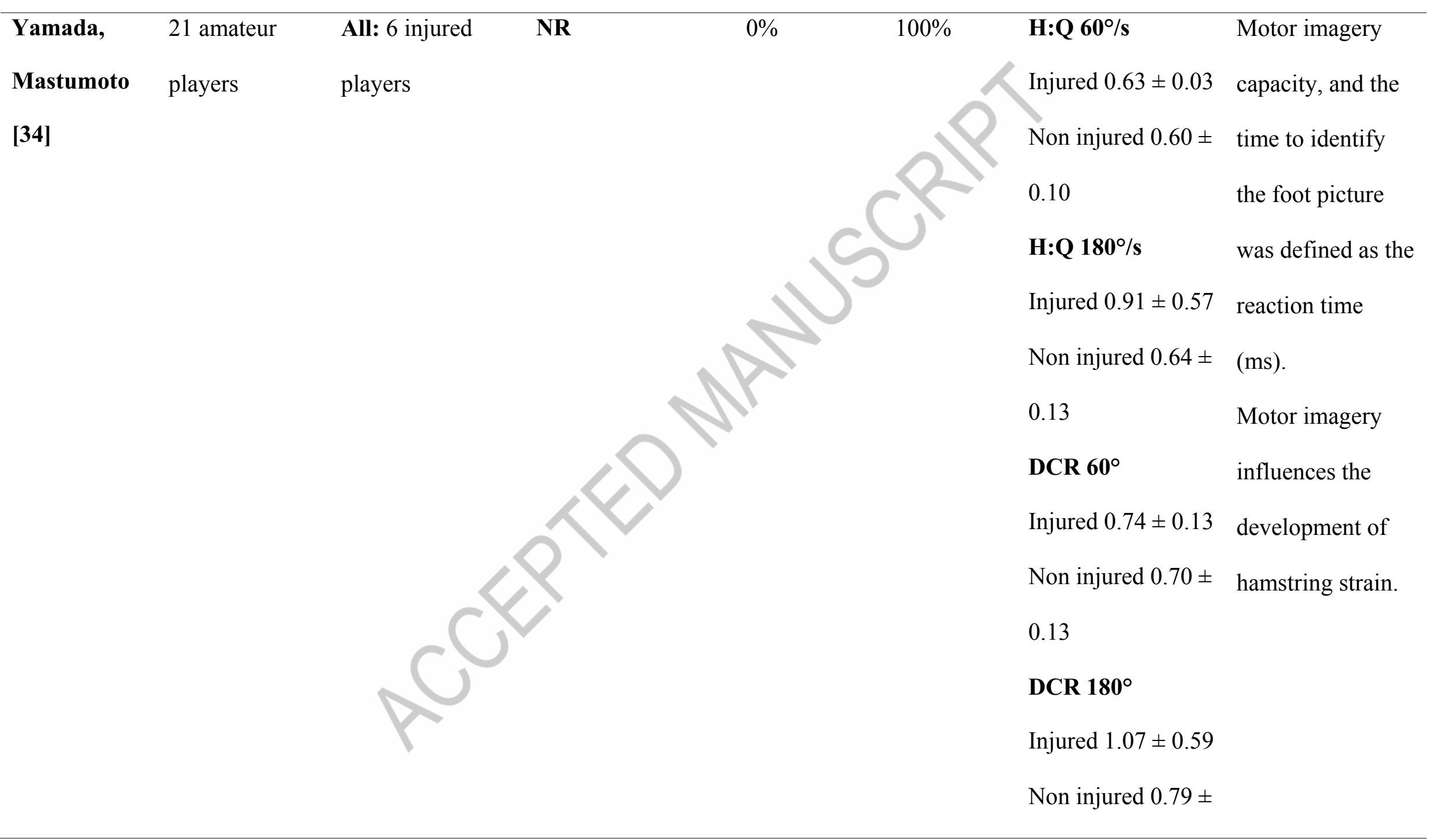




\subsection{6}

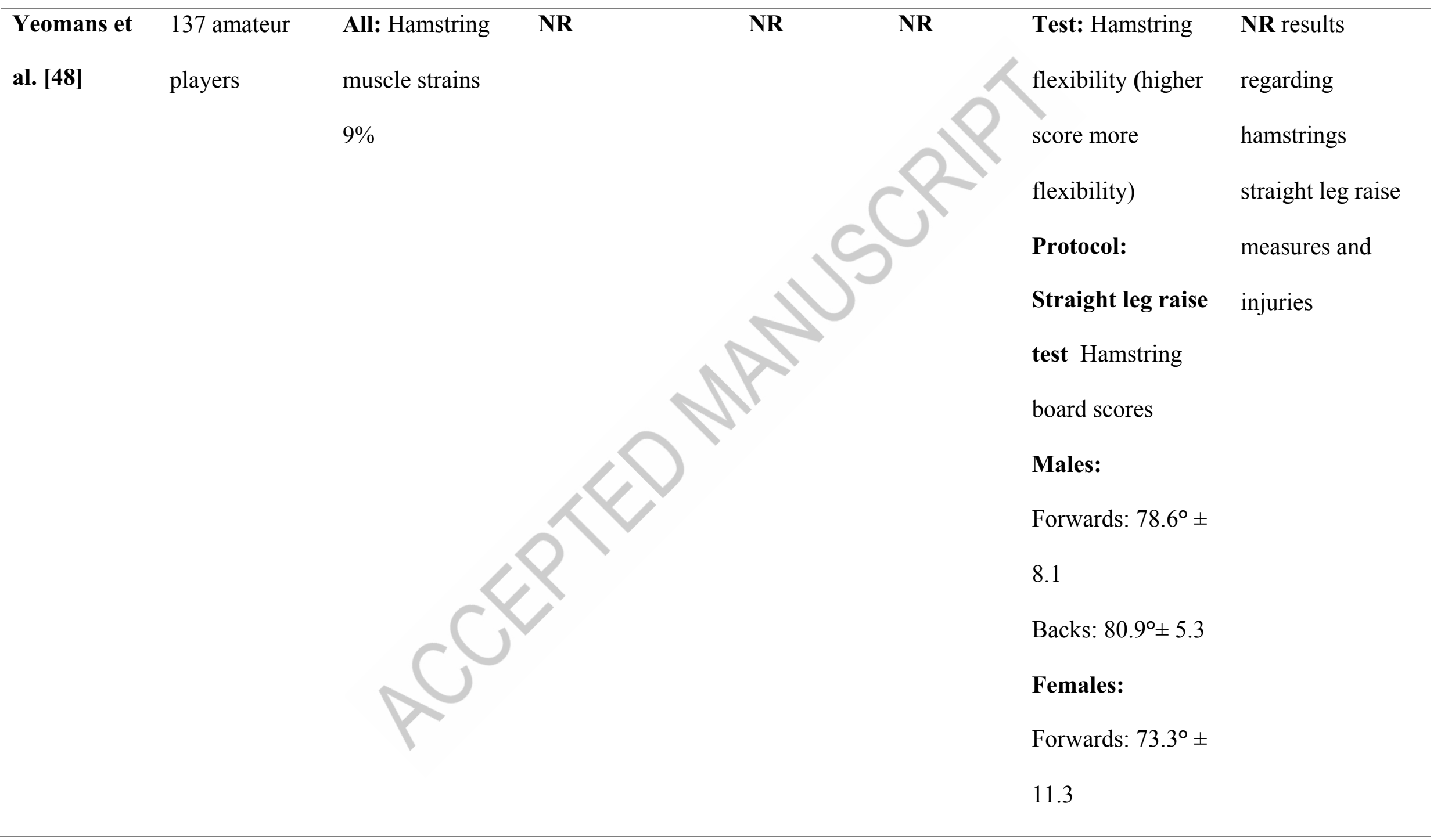




\section{1}

Abbreviations: AD, average distance; N Newton; NR, not reported; RTP, return to play.

Notes. Values are means \pm standard deviation, means [95\% confidence intervals], and minimum - maximum range.

Table 3. Hamstring strength measures and imbalances

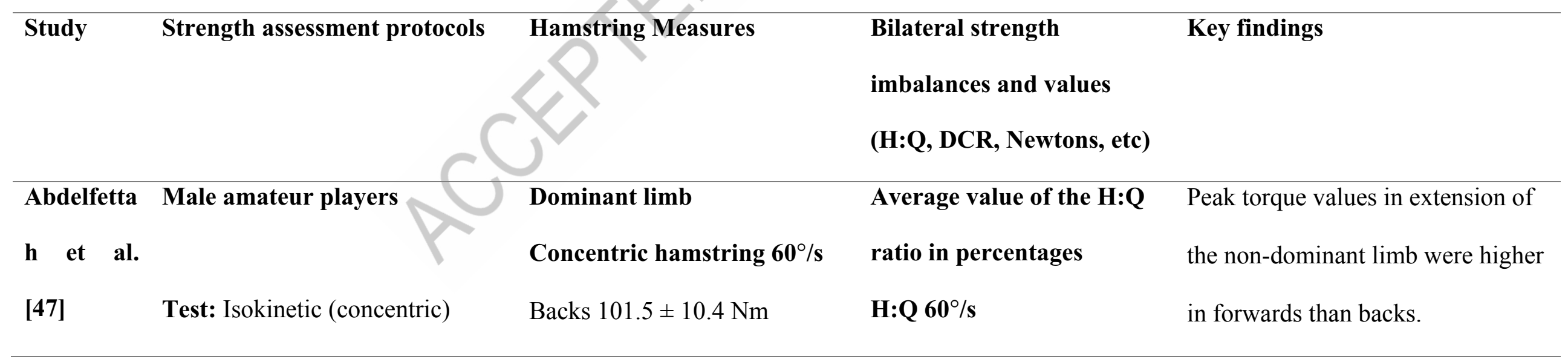




\begin{tabular}{|c|c|c|c|c|}
\hline & & Forwards $133.1 \pm 30.4 \mathrm{Nm}$ & Dominant limb & \\
\hline & Equipment: Cybex Norm & Concentric hamstring $180^{\circ} / \mathrm{s}$ & $56.4 \pm 9.5 \%$ & \\
\hline & isokinetic dynamometer & Backs $70.7 \pm 8.9 \mathrm{Nm}$ & Non dominant limb & \\
\hline & & Forwards $91 \pm 21.3 \mathrm{Nm}$ & $53.9 \pm$ & \\
\hline & Protocol: Peak 1RM at 60 and & Non dominant limb & & \\
\hline & $180^{\circ} / \mathrm{s}$ for series of successive & Concentric hamstring $60 \% / \mathrm{s}$ & $H: Q 180^{\circ} / \mathrm{s}$ & \\
\hline & movements of 5 and 30 & Backs $98 \pm 21.8 \mathrm{Nm}$ & nt $\operatorname{limb}$ & \\
\hline & repetitions. & Forwards & $56.1 \pm 10.25 \%$ & \\
\hline & & Concentric hamstring $180 \% / \mathrm{s}$ & Non dominant limb & \\
\hline & & Backs $72.7 \pm 15.6 \mathrm{Nm}$ & $56 \pm 6.6 \%$ & \\
\hline Anastasi, & Female amateur players & Dominant limb & Bilateral strength & Intervention group had a significant \\
\hline Hamzeh & & Pre-intervention & imbalances in percentage & decrease in the percentage of \\
\hline \multirow[t]{3}{*}{ [26] } & \multirow[t]{2}{*}{ Test: Isokinetic (concentric) } & $89.2 \pm 19.9 \mathrm{Nm}$ & & bilateral strength imbalances. \\
\hline & & Post intervention & Pre-training & \\
\hline & Equipment: Cybex Norm & $102.2 \pm 21.2 \mathrm{Nm}$ & $10.4 \pm 3.5 \%$ & \\
\hline
\end{tabular}


isokinetic dynamometer

Non dominant limb

Pre-intervention

Post-training

Protocol: Peak during pre- and

$93.15 \pm 20.0 \mathrm{Nm}$

$4.7 \pm 2.2 \%$

post-training in both legs at $60 \%$ Post intervention

$104.23 \pm 18.8 \mathrm{Nm}$

\section{Beyer et Male amateur players}

al. [31]

\section{Dominant leg}

Concentric hamstring $60 \%$

Inexperienced $114.3 \pm 4.8 \mathrm{Nm}$

Experienced $119.3 \pm 5.8 \mathrm{Nm}$

Concentric hamstring $180^{\circ} / \mathrm{s}$

Inexperienced $91.9 \pm 3.9 \mathrm{Nm}$

Experienced $92.8 \pm 4.7 \mathrm{Nm}$

Equipment: Biodex Medical

System isokinetic dynamometer

\section{Eccentric hamstring $60 \%$}

Inexperienced $207.3 \pm 8.2 \mathrm{Nm}$

Protocol: 10 experienced players

( $\geq 2$ years) and 14 inexperience
Experienced $225.8 \pm 9.9 \mathrm{Nm}$

Eccentric hamstring $180^{\circ} / \mathrm{s}$
H:Q 60\%

Inexperienced $0.45 \pm 0.02$

Experienced $0.50 \pm 0.02$

\section{H:Q $180^{\circ} / \mathrm{s}$}

Inexperienced $0.47 \pm 0.02$

Experienced $0.50 \pm 0.03$

DCR 60\%

Inexperienced $0.81 \pm 0.04$

Experienced $0.95 \pm 0.05$

\section{DCR $180^{\circ} / \mathrm{s}$}

Inexperienced $1.07 \pm 0.05$
Experience group had a

significantly greater DCR $60 \%$ s than the inexperienced group. No significant differences in mass, age, eccentric hamstring strength values at H:Q $60^{\circ} / \mathrm{s}, 180^{\circ} / \mathrm{s}$, or DCR $180^{\circ} / \mathrm{s}$ between groups. 
players $(<2$ years $)$.

Isokinetic: Peak of 3 RM

measured at $60^{\circ} / \mathrm{s}$ and $180^{\circ} / \mathrm{s}$

Isotonic: $1 \mathrm{RM}$ squat
Inexperienced $208.8 \pm 9.6 \mathrm{Nm} \quad$ Experienced $1.17 \pm 0.06$

Experienced $220.4 \pm 11.4 \mathrm{Nm}$

\section{Squat 1RM}

Inexperienced $132.4 \pm 9.5 \mathrm{~kg}$

Experienced $143.9 \pm 11.8 \mathrm{~kg}$

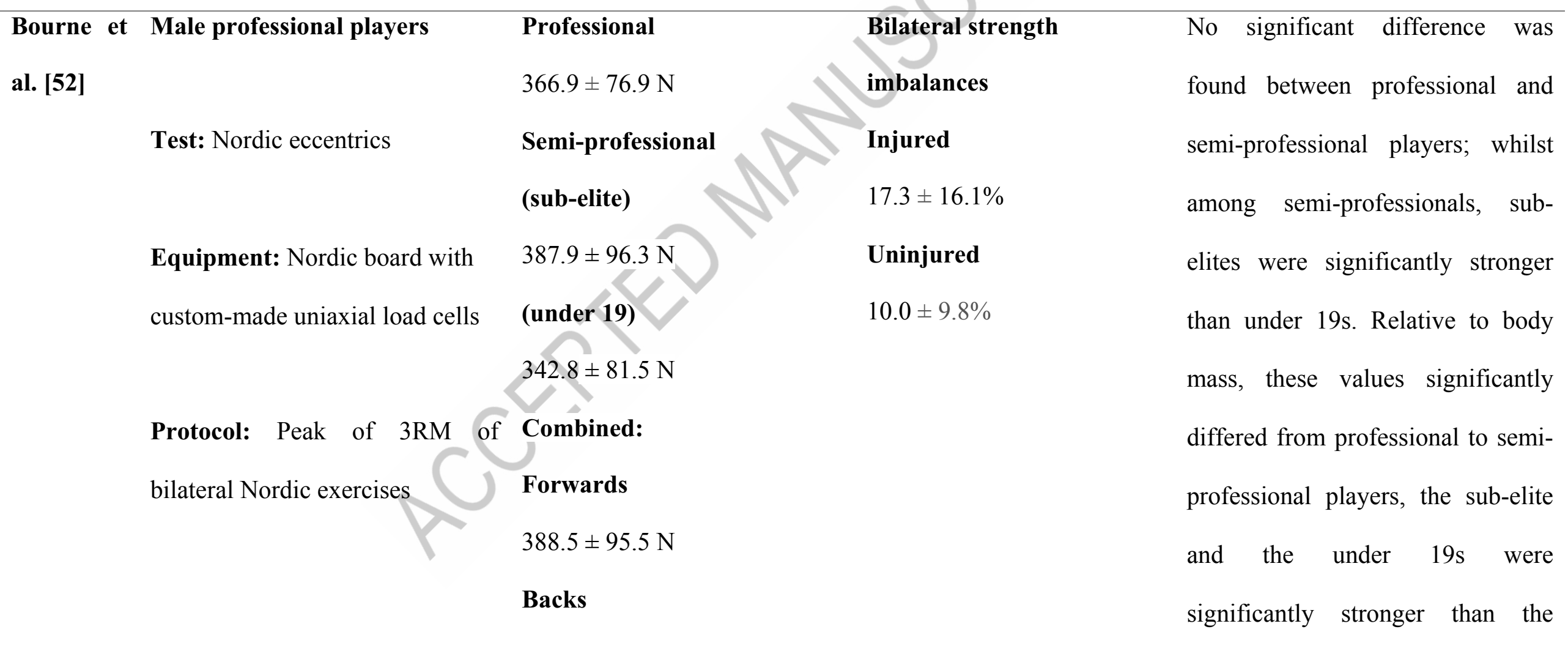


Forwards were stronger than backs; but relative to body weight, no significant differences were found.

Brown et Male professional players

al. [28]

Test: Isokinetic (concentric)

Equipment: Humac Norm

dynamometer

Protocol: Average peak torque

from $4 \mathrm{RM}$ measured at $60 \% \mathrm{~s}$

during seated knee-extension/

\section{Leg Extension}

Forwards

Dominant $281 \pm 45 \mathrm{Nm}$

Non-dominant $268 \pm 44 \mathrm{Nm}$

\section{Backs}

Dominant $244 \pm 29 \mathrm{Nm}$

Non-dominant $247 \pm 38 \mathrm{Nm}$

\section{Leg Flexion}

\section{Forwards}

Dominant $184 \pm 27 \mathrm{Nm}$

\section{H:Q}

Forwards

Dominant $0.66 \pm 0.09$

Non-dominant $0.68 \pm 0.10$

Backs

Dominant $0.64 \pm 0.10$

Non-dominant $0.64 \pm 0.08$ In two rugby codes, forwards were taller and heavier than backs.

Professional Rugby Union

forwards had significantly larger peak torque during knee flexion in both dominant and non-dominant limbs compared to professional rugby league forwards and Rugby Union backs. 
flexion and supine hip-

extension/flexion at $60 \%$ s.

Dominant $157 \pm 27 \mathrm{Nm}$
Non-dominant $156 \pm 27 \mathrm{Nm}$

Brown et Male amateur players

al. [30]

Test: Isokinetic (concentric and

Forwards

Dominant $252 \pm 62 \mathrm{Nm}$

Non-dominant $228 \pm 38 \mathrm{Nm}$

\section{Backs}

Dominant $225 \pm 38 \mathrm{Nm}$

Non-dominant $214 \pm 53 \mathrm{Nm}$

Leg Flexion Concentric

Protocol: 1 RM with the

dominant and nondominant limb

in sitting and in a supine position

at $60 \%$ s. Dominant limb was

\section{Forwards}

Dominant $129 \pm 25 \mathrm{Nm}$

Non-dominant $124 \pm 19 \mathrm{Nm}$

Backs
H:Q

Forwards

Dominant 0.52

Non-dominant 0.55

\section{Backs}

Dominant 0.52

Non-dominant 0.56

DCR

Forwards

Dominant 0.65

Non-dominant 0.64

Backs
Professionals were stronger in all

the peak torque measures compared to academy players.

In forwards, the dominant limb was stronger than the non-dominant

limb.

The average $\mathrm{H}: \mathrm{Q}$ ratio was less than 0.6 and the DCR was more than 0.6. In the isokinetic eccentric knee strength test, results showed forwards were stronger in the dominant leg compared to the non- 


\begin{tabular}{|c|c|c|c|c|}
\hline \multirow[t]{11}{*}{ 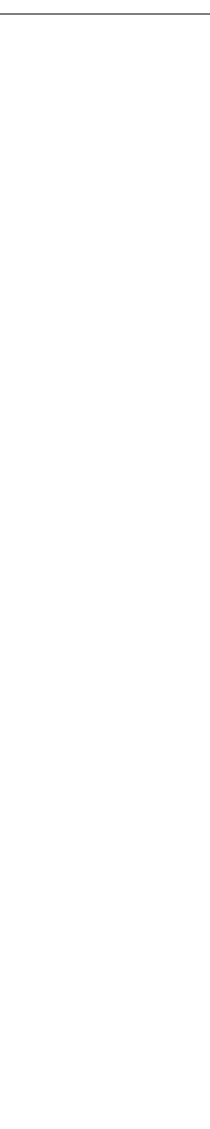 } & defined as the limb that the player & Dominant $115 \pm 14 \mathrm{Nm}$ & Dominant 0.65 & dominant leg. Backs had similar \\
\hline & preferred to kick the ball or could & Non-dominant $118 \pm 28 \mathrm{Nm}$ & Non-dominant 0.66 & strength torque values in both legs \\
\hline & kick the ball the farthest. & Leg Flexion Eccentric & & in flexion, however the dominant \\
\hline & & Backs & & leg was stronger in extension. No \\
\hline & & Dominant $148 \mathrm{Nm}$ & & differences between positions and \\
\hline & & Non-dominant $125 \mathrm{Nm}$ & & limbs were found. Isokinetic \\
\hline & & Forwards & & concentric hip strength in forwards \\
\hline & & Dominant $155 \mathrm{Nm}$ & & were similar between dominant and \\
\hline & & Non-dominant 14 & & non-dominant legs. Forwards \\
\hline & & & & displayed greater values in the \\
\hline & & & & dominant leg during flexion \\
\hline \multirow{4}{*}{$\begin{array}{l}\text { Deighan } \\
\text { et al. [29] }\end{array}$} & Male amateur players & Seated peak torque & $\mathbf{H : Q}$ & In a seated position, concentric \\
\hline & & Hamstring concentric & Seated & peak torque was greater compared \\
\hline & Test: Isokinetic (Concentric) & $60^{\circ} / \mathrm{s}: 144 \pm 26 \mathrm{Nm}$ & $60^{\circ} / \mathrm{s}: 0.53 \pm 0.07$ & to supine eccentric. The $H: Q$ in the \\
\hline & & $180^{\circ} / \mathrm{s}: 121 \pm 16 \mathrm{Nm}$ & $180^{\circ} / \mathrm{s}: 0.56 \pm 0.07$ & seated position showed no \\
\hline
\end{tabular}




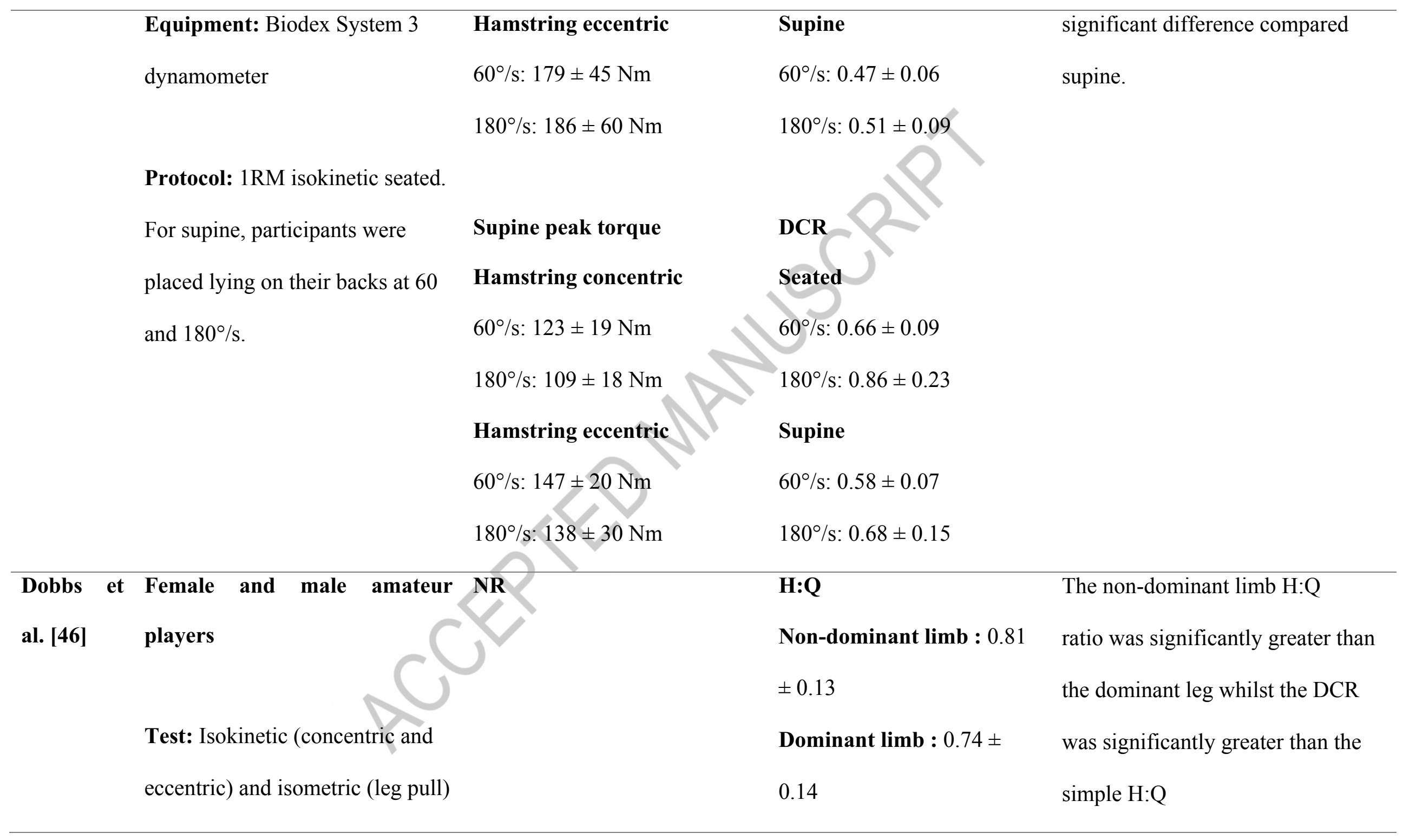


Equipment: Biodex Pro 4

dynamometer

\section{Protocol:}

Isokinetic tests: Average of 3RM

at $60 \%$ s; $1 \mathrm{RM}$ isometric

quadriceps strength at $60^{\circ} \mathrm{s}$, and

$1 \mathrm{RM}$ isometric hamstring

strength at $35 \% \mathrm{~s}$

Isometric: Bilateral and

unilateral leg isometric mid-thigh pull. 3RM for both legs, and 2RM

for a single leg were recorded on

force plates.

Dominant limb was defined as

the preferred leg to kick a ball.
$0.81 \pm 0.14$

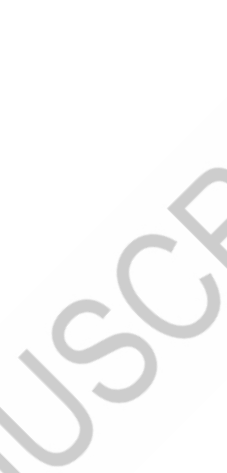


Mondin et Male professional players

\section{NR}

al. [36]

Test: Sphygmomanometer

measures of maximal isometric

strength. Isokinetic dynamometry

(concentric) strength.

Equipment: Humac Norm

dynamometer and an adapted

sphygmomanometer

Protocol:

Isokinetic tests: Peak of 3RM at

$60 \%$ s.

The sphygmomanometer test:

Subjects in supine with knees
$\operatorname{ICC}(95 \% \mathrm{CI})$ :

Quadriceps $90^{\circ}$ right

$0.64(-0.28-0.91)$

Quadriceps $90^{\circ}$ left

$0.81(0.21-0.95)$

Hamstrings $90^{\circ}$ right

$0.83(0.30-0.96)$

Hamstrings $90^{\circ}$ left

$0.87(0.45-0.97)$

Hamstrings $30^{\circ}$ right

$0.92(0.69-0.98)$

Hamstrings $30^{\circ}$ left

$0.87 \quad(0.45-0.97)$
A positive correlation in $90^{\circ}$ of

knee flexion between

sphygmomanometer and isokinetic

tests was found, as well as

hamstring strength at $90^{\circ}$ and $30^{\circ}$

of knee flexion for both measures.

No relation in strength asymmetry

between legs or tests at $30^{\circ}$ or $90^{\circ}$

when testing the efficacy of the

sphygmomanometer compared to

the isokinetic test. No correlation in

hamstring to quadriceps ratio at $90^{\circ}$

between test for dominant and non-

dominant leg.

The group found reliability and 
flexed at $30^{\circ}$ or $90^{\circ}$ and heel of

one leg on the cuff, and opposite

leg resting on the floor and

extended. 3RM isometric strength

of hamstring at $30^{\circ}$ and $90^{\circ}$ were

recorded.

\section{Severo- Male professional players}

\section{Silveira et}

al. [41]

\section{Test: Isokinetic (concentric and} eccentric)

Equipment: ultrasound

assessment B-mode

ultrasonography and Biodex Pro

4 dynamometer
$\mathrm{Nm}$

Progressive group: $278 \pm 48.8$

$\mathrm{Nm}$

\section{Pre-intervention}

\section{Quadriceps concentric}

Constant group: $275.5 \pm 27.1$

\section{Hamstrings concentric}

Constant group: $142.2 \pm 19.6$

$\mathrm{Nm}$

Progressive group: $146.6 \pm$

validity measuring hamstring

strength with the adapted

sphygmomanometer test, although

the test was not valid or reliable

assessing bilateral or hamstrings to

quadriceps asymmetries.

Pre-intervention

H:Q

Constant group: $0.52 \pm 0.05$

Progressive group: $0.53 \pm$

0.07

DCR

Constant group: $0.74 \pm 0.14$

Progressive group: $0.76 \pm$

0.06
After the training intervention only

the progressive group had an

increased in hamstring concentric

and eccentric peak torques, $\mathrm{H}: \mathrm{Q}$

and DCR. 
Protocol: Peak of $3 \mathrm{RM}$ of $24.3 \mathrm{Nm}$

flexion-extension at $60^{\circ} / \mathrm{s}$

Hamstrings eccentric

Constant group $204.5 \pm 43.3$

$\mathrm{Nm}$

Progressive group $211.1 \pm 31.8$

$\mathrm{Nm}$

Notes. Values are means \pm standard deviations.

Abbreviations: DCR, isokinetic dynamic control ratio; H:Q, isokinetic hamstring quadriceps ratio; Nm, Nanometre; NR, not reported; RM, repetition maximum. 
Table 4. Hamstring training protocols and results

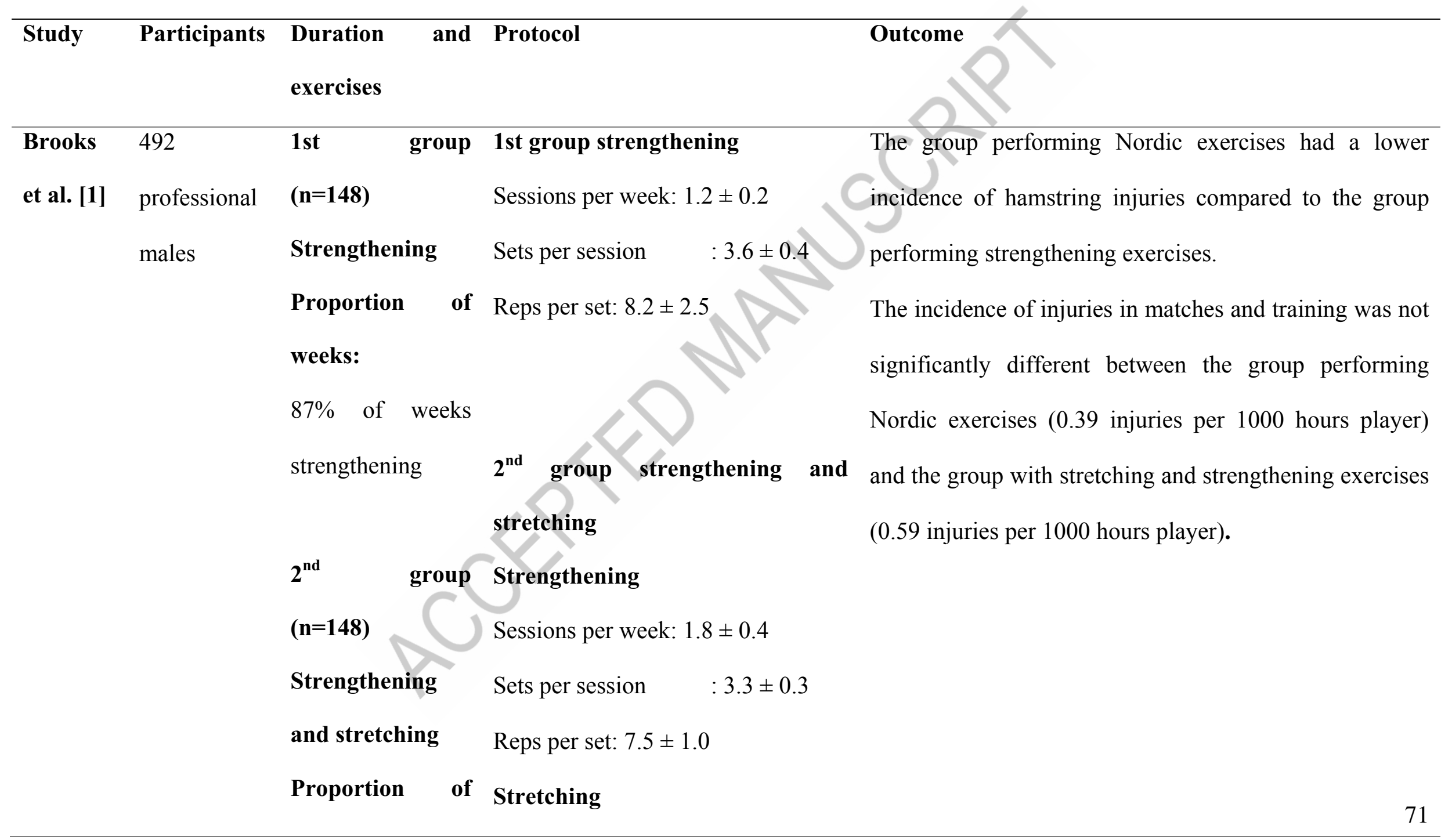


strengthening and Reps per set: $2.5 \pm 9.0$

$87 \%$ of weeks

stretching $\quad \mathbf{3}^{\text {rd }}$ group strengthening stretching

and Nordic exercises

Strengthening

Sessions per week: $1.3 \pm 0.3$

$3^{\text {rd }}$

group Sets per session $\quad: 3.0 \pm 0.4$

$(\mathbf{n}=\mathbf{2 0 0})$

Reps per set: $7.5 \pm 2.1$

Proportion

of Stretching

weeks:

Sessions per week: $1.8 \pm 0.2$

$44 \%$ of weeks Sets per session $\quad: 2.6 \pm 0.4$

strengthening, Reps per set: $28 \pm 20$

$87 \%$ of weeks Nordic exercises

stretching, and Sessions per week: $1.3 \pm 0.5$ 


$\begin{array}{lll}65 \% \text { of weeks } & \text { Sets per session } & : 2.8 \pm 0.7 \\ \text { Nordic exercises } & \text { Reps per set } & 6.7 \pm 1.5\end{array}$

\begin{tabular}{|c|c|c|c|c|}
\hline & 24 females & 10 weeks & \multirow{2}{*}{$\begin{array}{l}\text { Program } \\
\text { Weeks } 1-2: 3 \text { sets x } 6 \text { reps }\end{array}$} & \multirow{3}{*}{$\begin{array}{l}\text { The } 10 \text {-week training program significantly decreased } \\
\text { the bilateral strength imbalances from: } 10.38 \pm 3.53 \% \text { to }\end{array}$} \\
\hline & \multirow{2}{*}{$\begin{array}{l}\text { amateur }(11 \\
\text { tested }\end{array}$} & Nordic exercises & & \\
\hline & & performed & Weeks $3-4: 3$ sets $\times 7$ rep & \\
\hline \multirow{3}{*}{$\begin{array}{l}\text { Anastasi, } \\
\text { Hamzeh } \\
{[26]}\end{array}$} & $\begin{array}{l}\text { players, } 1 \\
\text { control } \\
\text { players) }\end{array}$ & couples & Weeks $5-7: 3$ sets $x$ & The Nordic exercise group displayed a significant change \\
\hline & control & $\begin{array}{l}\text { players, } 3 \text { times } \\
\text { per week }\end{array}$ & Weeks $8-10: 3$ sets $x 10$ rep & in the mean maximal vertical jump height from 31.22 to \\
\hline & players) & \multicolumn{2}{|r|}{$N$} & $35.93 \mathrm{~cm}$ \\
\hline
\end{tabular}

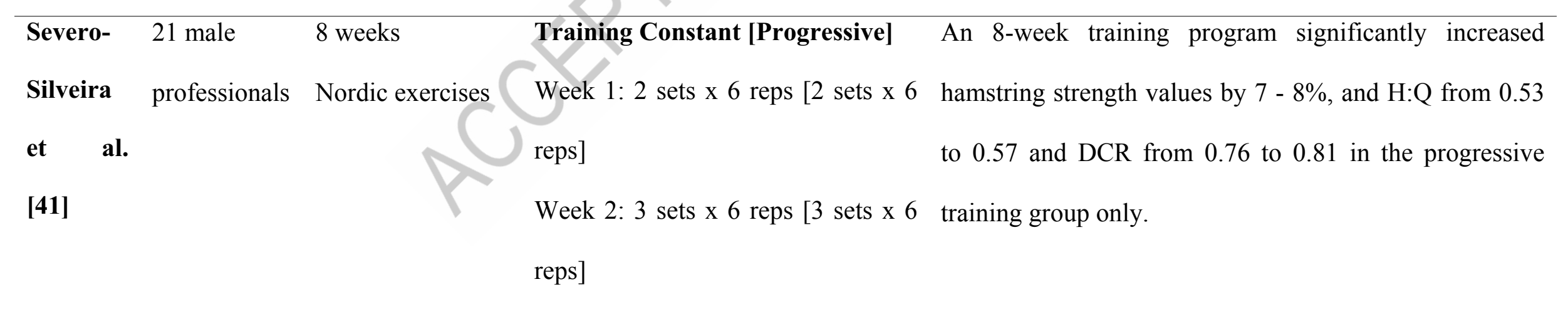


Week 3: 3 sets $\times 6$ reps [3 sets $\times 8$

reps]

Week 4: 3 sets $\times 6$ reps [3 sets $\times 10$

reps]

Week 5: 3 sets $\times 6$ reps [4 sets $x$ 8-

10 reps]

Week 6: 3 sets X 6 reps [4 sets $\times$ 8-

10 reps]

Week 7: 3 sets X 6 reps [4 sets X 10

reps]

Week 8: 3 sets X 6 reps [4 sets x 10

reps]

Notes. Values are means \pm standard deviation.

Abbreviations: DCR, dynamic ratio; $\mathrm{H}: \mathrm{Q}$ Hamstring to quadriceps ratio 


\section{Figures}

Identification of new studies via databases and registers
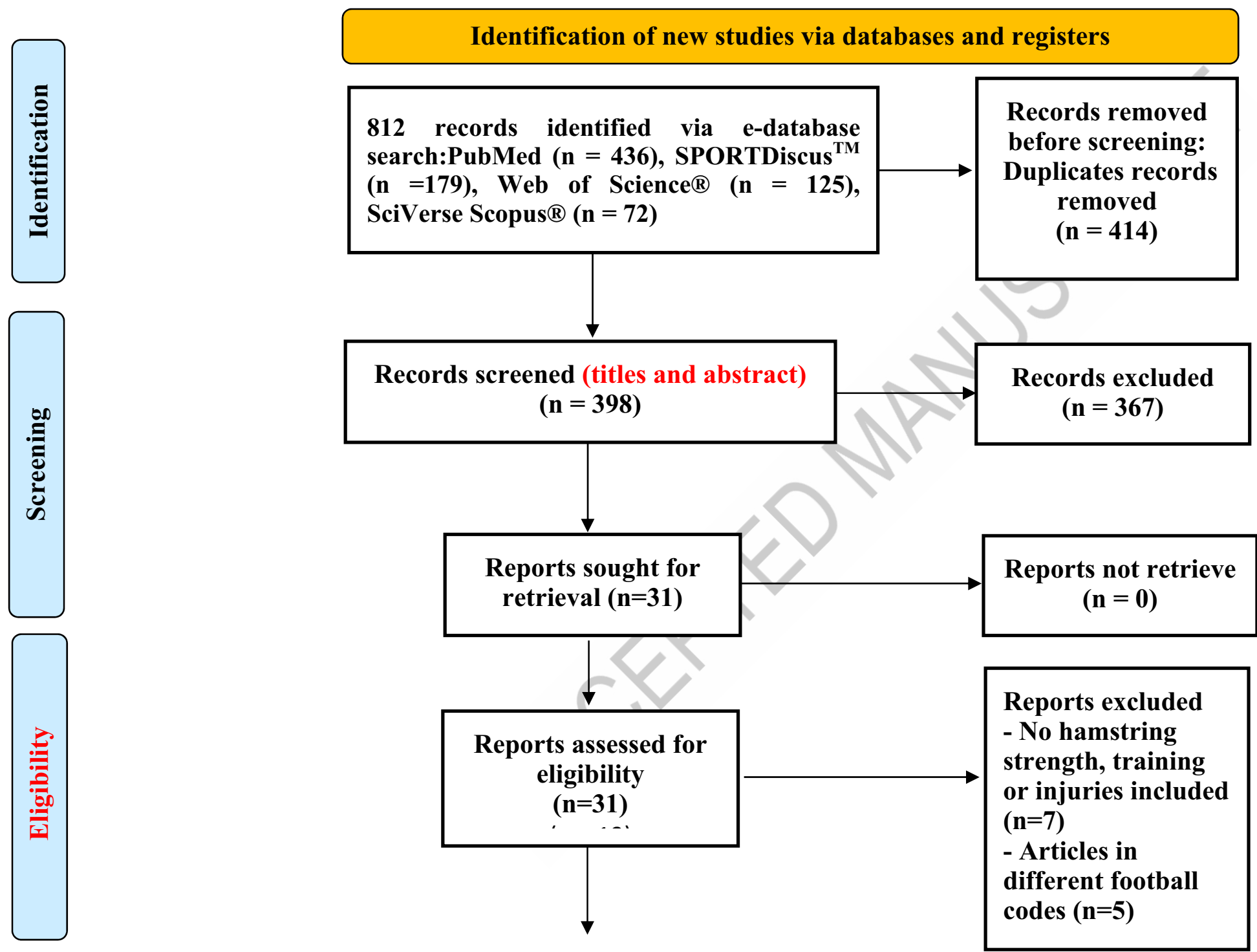

Identification of new studies via other

\begin{tabular}{|c|}
\hline New studies included \\
in review: \\
Google scholar \\
search $(\mathrm{n}=2)$ \\
Citation search \\
$(\mathrm{n}=3)$
\end{tabular}



in review $(n=24)$

Figure 1. Preferred Reporting Items for Systematic Reviews and Meta-Analyses flow chart. 


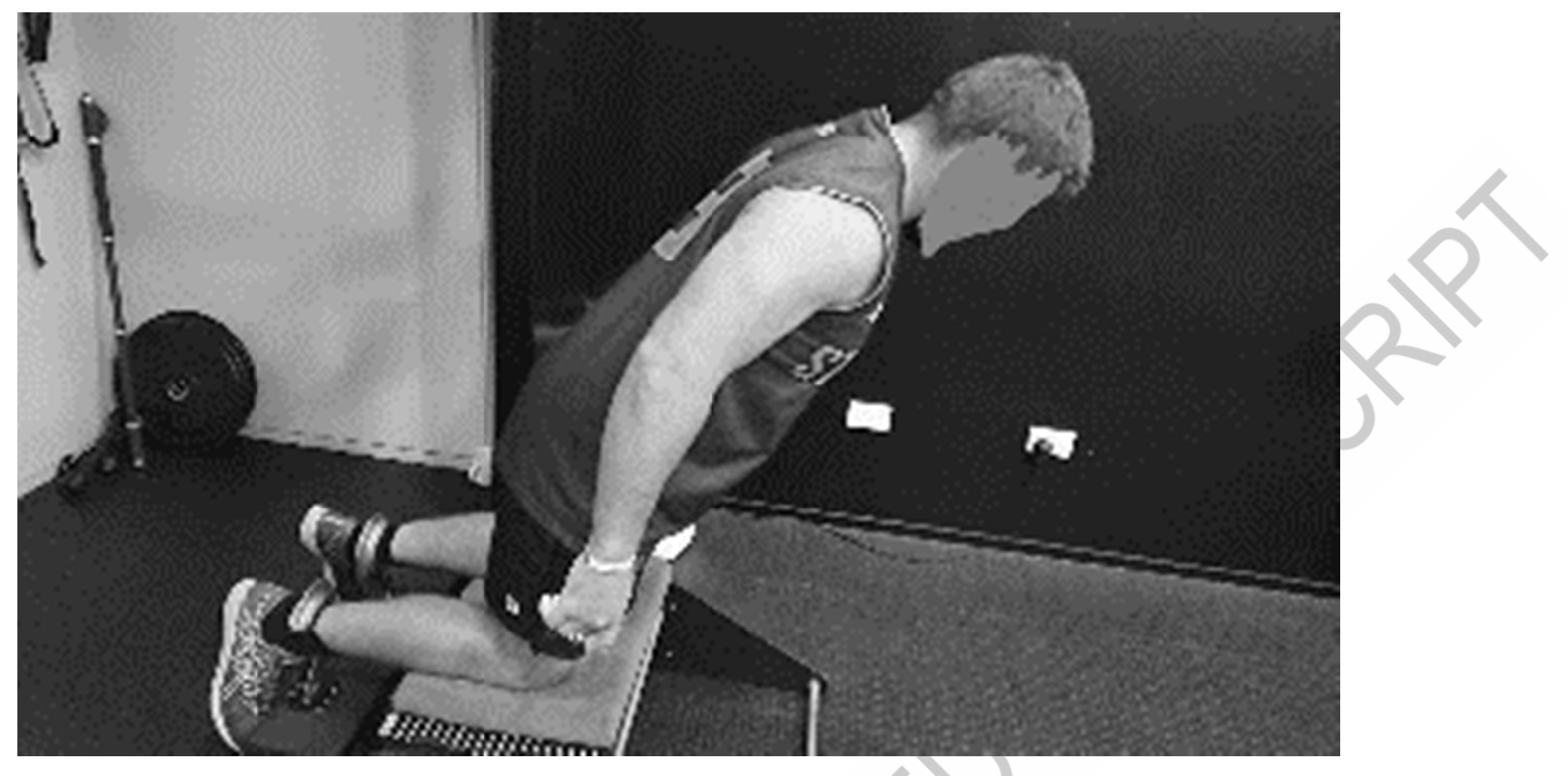

Figure 2. Illustration of a Nordic hamstring exercise. 


\section{Supplemental material}

\section{Appendix 1}

Quality assessment tool

\section{NEWCASTLE - OTTAWA QUALITY ASSESSMENT SCALE}

Selection: (Maximum 5 stars)

1) Representativeness of the sample:

a) Truly representative of the average in the target population. * (all subjects or

random sampling

b) Somewhat representative of the average in the target population. * (non-

random sampling)

c) Selected group of users.

d) No description of the sampling strategy.

2) Sample size:

a) Justified and satisfactory. *

b) Not justified. 
3) Non-respondents: Rate between participants asked to participate and participants actually participated

a) Comparability between respondents and non-respondents characteristics is established, and the response rate is satisfactory. * Rate more than $60 \%$

b) The response rate is unsatisfactory, or the comparability between respondents and non-respondents is unsatisfactory.

c) No description of the response rate or the characteristics of the responders and the non-responders.

4) Ascertainment of the exposure (risk factor):

a) Validated measurement tool. *

b) Non-validated measurement tool, but the tool is available or described.*

c) No description of the measurement tool.

\section{Comparability: (Maximum 2 stars)}

1) The subjects in different outcome groups are comparable, based on the study design or analysis. Confounding factors are controlled.

a) The study controls for the most important factor (select one). * Age 
b) The study control for any additional factor. * Weight , height

\section{Outcome: (Maximum 3 stars)}

1) Assessment of the outcome:

a) Independent blind assessment. **

b) Record linkage. **

c) Self report. *

d) No description.

2) Statistical test:

a) The statistical test used to analyze the data is clearly described and

appropriate, and the measurement of the association is presented, including

confidence intervals and the probability level ( $\mathrm{p}$ value). *

b) The statistical test is not appropriate, not described or incomplete.

Table 5. Summary of the risk of bias assessment of the reviewed studies (Modified Newcastle - Ottawa Quality score). 


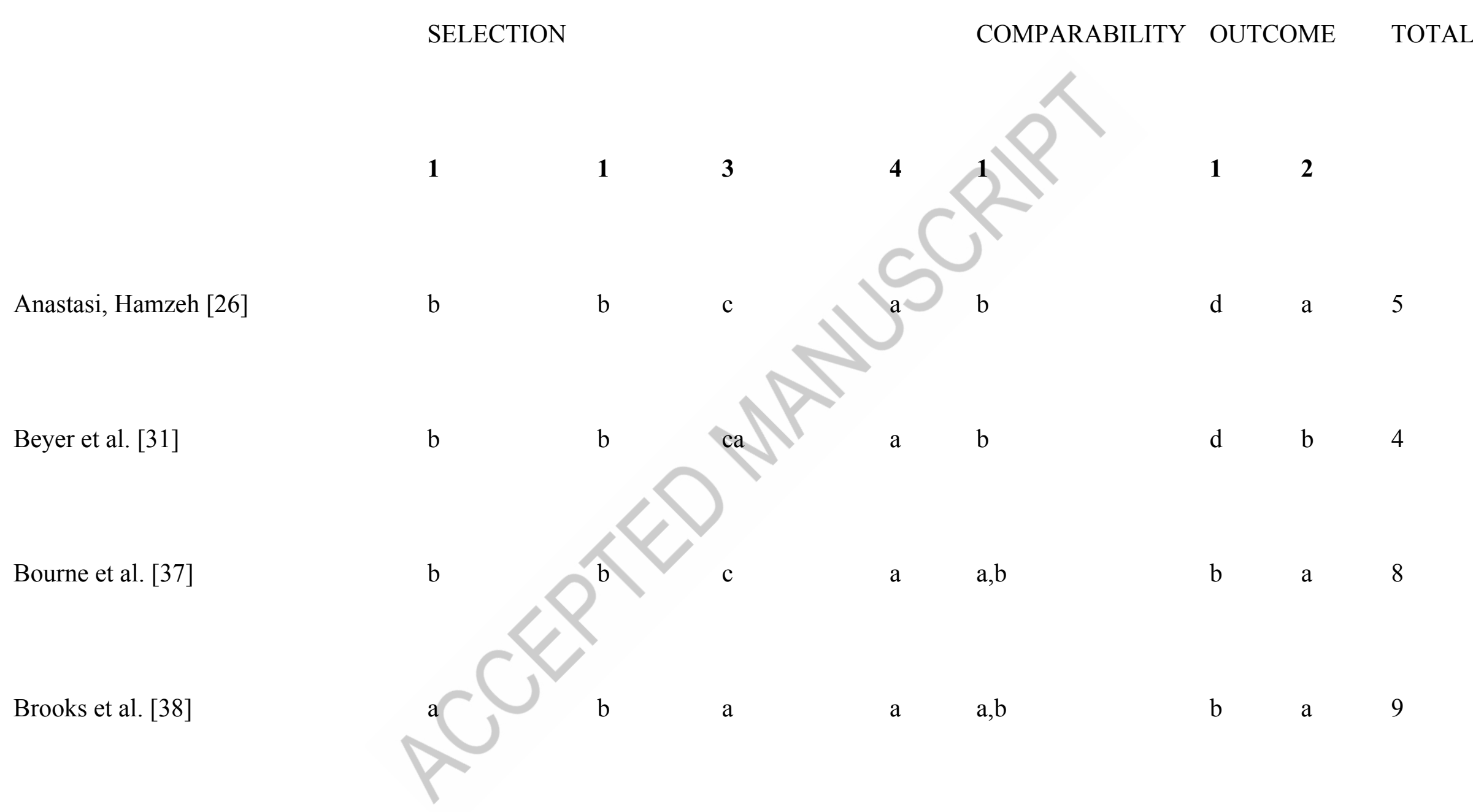


Brooks et al. [39]

Brooks et al. [69]

Brooks, Kemp [43]

Brown et al. [28]

Brown et al. [30]

Deighan et al. [29]

[46] b $\quad$ a $\quad 9$

b

a

b

c

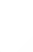

b

c

b a,b

b b

b b $\begin{array}{lll}\text { b } & \text { a } & 9\end{array}$

$\begin{array}{lll}\text { b } & \text { a } & 7\end{array}$

$\begin{array}{lll}\mathrm{d} & \mathrm{B} & 4\end{array}$

$\begin{array}{lll}\mathrm{d} & \mathrm{b} & 4\end{array}$

$\begin{array}{lll}\mathrm{d} & \mathrm{a} & 4\end{array}$

D $\quad$ b 2 
Farnan et al. [32]

Kenneally-Dabrowski et al. [45]

Kenneally-Dabrowski et al. [44]

Mendiguchia et al. [33]

Mondin et al. [36]

Reid et al. [27]

Roberts et al. [42] b

c

b

c

b

c

c

(1) b

b

a b

a a,b

a b

c a a,b

b c c a

5

$\begin{array}{lll}\mathrm{d} & \mathrm{b} & 4\end{array}$

d $\quad$ a $\quad 4$

b $\quad$ b $\quad 6$
a

b

$\begin{array}{llll}\text { b } & \text { a } & 7\end{array}$


Severo-Silveira et al. [41]

Upton et al. [35]

Yamada, Mastumoto [34]

b

b

b

b b

a

b

b

b c

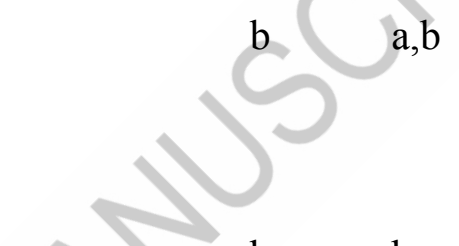

a

8

b $\begin{array}{lll}\mathrm{a} & \mathrm{b} & 7\end{array}$

b

5

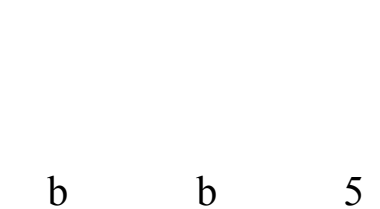

Methodology quality assessment score based on Newcastle-Ottawa Quality Assessment Scale adapted for cross-sectional studies, weak (0-3 stars), moderate (4-6 stars), and strong (7-10 stars). 